\title{
ÉMERGENCES DE LA PHILOSOPHIE AU MOYEN ÂGE *
}

S'interroger sur l'apparition de la philosophie médiévale peut sembler une entreprise vouée à l'échec. La moindre des difficultés vient de la diversité des aires géographiques et spirituelles où elle s'est développée : le christianisme - grec et latin - l' lislam, le judaïsme. On peut la résoudre en la divisant en ses parties. Bien plus redoutable est la question que pose le mot philosophie, et plus précisément la légitimité de son emploi quand il s'agit de la pensée médiévale. Partout animée de représentations religieuses fortes, surveillée par les institutions chargées de les maintenir, est-elle d'un aloi philosophique assez indiscutable pour qu'on puisse valablement la comparer à celle d'autres époques, notamment l'Antiquité et la Renaissance ? À ce soupçon, issu d'une conception traditionnellement rationaliste de la philosophie, fait écho dans l'historiographie le débat autour de la " philosophie chrétienne " qui, amorcé depuis plus d'un siècle, s'est développé il y a environ cinquante ans entre des philosophes chrétiens eux-mêmes pour la plupart ${ }^{1}$. Deux questions y confluent : peut-on, par une manière d'analyse conceptuelle, isoler complètement dans les synthèses scolastiques leur composante philosophique ? C'est là une question historique ; l'autre est doctrinale : peut-on ou non appeler philosophes des penseurs selon qui la réflexion proprement humaine doit rechercher, ou simplement accueillir, des données et des suggestions issues d'une foi religieuse ? Il est clair que ces problèmes préjudiciels sup-

* Cet article était entièrement écrit quand j'ai pu lire La Philosophie au Moyen Âge, de Lambert-Marie DE RIJK (trad. franç. de Pierre SwIGgers, Leiden, Brill, 1985 ; le texte néerlandais original avait paru en 1981). J'ai eu le plaisir de constater que, sans concertation, nos vues sur les débuts de la philosophie médiévale étaient fort voisines, pour ne pas dire identiques: voir notamment op. laud., p. 70 et surtout p. 77.

1. Voir les abondantes " notes bibliographiques " fournies à ce sujet par Étienne GILSON, L'Esprit de la philosophie médiévale, Paris, Vrin, $2^{e}$ éd., 1944, p. 413-440.

Revue de synthèse : IV" S. Nos 3-4, juil.-déc. 1987 
posent d'une manière ou d'une autre une conception génériquement commune de la philosophie, qui, démarche rationnelle sans compromission, commencée et poursuivie dans un champ strictement défini, serait en droit assez autonome pour qu'on puisse la concevoir en sa pureté. Les questions apparemment historiques s'avèrent donc dogmatiques, si bien qu'au lieu de nous lier, elles nous libèrent : nous voici amenés, par nécessité de méthode, à considérer empiriquement comme étant philosophie, 1) ce qu'ont nommé ainsi et exposé des hommes qui se donnaient et qu'on reconnaissait comme des philosophes, et 2) des ensembles doctrinaux qui, sans porter le nom de philosophie, voire en le refusant, ont assez d'affinités avec ceux du premier groupe pour qu'on soit autorisé à le leur attribuer pourtant. Nous allons donc examiner d'abord comment la philosophie, ainsi définie, du Moyen Âge chrétien d'Occident se situe par rapport à celle de l'Antiquité païenne, quant à sa forme et quant à son contenu. Précisons que, lorsque nous parlons du Moyen Âge, nous n'entendons par-là qu'un cadre purement chronologique : la formule ne préjuge en rien de la datation de la philosophie médiévale - puisque justement il s'agit d'en déterminer la naissance.

\section{I. - QUAND LE MOYEN ÂGE LATIN EUT-IL SA PHILOSOPHIE ?}

Ce qui frappe d'emblée quand on compare la pensée médiévale à l'antique, selon les images les plus vives qui viennent aussitôt à l'esprit, c'est la différence de leurs contenus religieux, assez grande pour cacher le reste. Le Moyen Âge a rempli les bibliothèques de traités des choses saintes, qui ne représentent qu'une faible part de ce qu'en ont renvoyé les murs des écoles. Un Dieu trine, l'incarnation du Fils, l'histoire collective d'un salut vers lequel cheminent les élus, rien de tout cela, pris à la rigueur, n'a de sens dans la sphère de la philosophie antique. Les textes révélés sur lesquels se fondent ces conceptions, le contenu spéculatif et normatif qui y est exposé ou qui peut s'en déduire, ont suscité une infinité de travaux théoriques où l'on peut voir la véritable philosophie du Moyen Âge. Si l'on prend ainsi les choses, tout est dit : de l'esprit antique au médiéval il n'y a qu'altérité radicale, comme l'exprime déjà l'Épître aux Romains. À la rupture ainsi proclamée par Paul font écho, parmi d'autres, les refus d'un Pierre de Celle : « les philosophes tissent des toiles d'araignées ", d'un Bernard de Clairvaux : «leur chef est Béhémoth "².

2. Pierre de Celle, L'école du cloître, éd, et trad. Gérard de Martel, Paris, Cerf ("Sources chrétiennes ", 240), 1977, p. 176 et suiv.; Bernard DE Clairvaux, 8 e Sermon sur le Cantique des Cantiques. 
Soit. Mais cette vue des choses ne nous instruit guère sur leur histoire, et si l'on veut comparer les contenus spirituels il vaut mieux ne pas les prendre tels que les présentent ceux qui en ont vécu le plus intensément les différences : on ne relèvera sinon qu'oppositions, ou du moins incommunication. Au surplus, tous les Médiévaux n'ont pas parlé sur le même ton que les mystiques qu'on vient d'entendre : à l'opposé on peut citer, en ce même XII siècle, Adélard de Bath, qui développe en pur philosophe et en pur physicien ses Questions naturelles ${ }^{3}$, et Bernard Silvestre, dont la Cosmographia offre une cosmogonie et une cosmologie où les éléments chrétiens apparaissent à peine ; et encore, à partir du XIII' siècle, le courant averroïste, dont l'histoire est celle d'un progrès continu vers l'incroyance. Entre ces extrêmes, on repérera l'aristotélisme des scolastiques classiques ${ }^{4}$, et, situé différemment, Pierre Abélard qui, méditant sur les trois personnages pauliniens : le Gentil, le Juif, le Chrétien, voire les mettant en scène, rapproche le Philosophe du Chrétien autant qu'il est possible et du texte même de Paul tire une apologie des philosophes ${ }^{5}$. Ces gens ne se sentaient donc pas si étrangers aux philosophes antiques, tout en mesurant différemment l'intervalle qui les en séparait ; sauf parti pris, ce ne sont pas des témoins qu'on puisse récuser. Mais encore, si Aristote reste loin de Paul, comme le dit, précisément, Abélard lui-même, Épicure, les Cyniques, sont-ils proches de Platon ? Si l'on compare deux à deux les religiosités platonicienne, épicurienne, chrétienne, entre lesquelles mettra-t-on la plus grande distance?

Une fois admis cependant que les contenus spirituels, pour autant qu'on en peut dégager l'essence, isolent finalement chaque philosophie dans sa sphère, il faut ajouter qu'inversement l'histoire les fait communiquer. On en trouvera la meilleure preuve peut-être en examinant un thème dont É. Gilson fait le point original et indépassable de la pensée médiévale latine : la distinction de l'être et de l'étant, inspirée ou mieux révélée par l'énoncé même du Nom divin entendu par Moïse (Exode, 3, 14) et formulée philosophiquement par Thomas d'Aquin - « ce pur Exister que saint Thomas philosophe rencontrait au terme de la métaphysique [et que] saint Thomas théologien... rencontrait aussi dans l'Écriture " 6 , conduisant ainsi « la philosophie dans ses voies nouvelles " ${ }^{7}$; car, dit encore

3. Jean Jolivet, "Adélard de Bath ou la nature sans le Livre ", Mélanges René Crozet, Poitiers, Société d'études médiévales, 1966, p. 93-99.

4. Certes avec beaucoup de nuances; mais on veut seulement noter ici, entre le christianisme et la philosophie, quelques accords, ou collusions, ou mariages forcés, comme chacun pourra dire à son gré, et d'abord selon les cas.

5. Commentaire de l'Épître aux Romains, PL 178, 802-805.

6. É. Gilson, Le Thomisme, Paris, Vrin, Se éd., 1945, p. 136.

7. ID., L'Être et l'Essence, Paris, Vrin, 1948, p. 84. 
Gilson, « la jonction de la métaphysique de l'être et de la théologie de l'Exode a mis des siècles à s'opérer ${ }^{8}$. Dans la mesure où l'éminent historien suggère que saint Thomas a fait faire ici à la philosophie un saut qualitatif, tout en marquant ce qui dans les siècles antérieurs pouvait préparer cet événement doctrinal, il considère que c'était bien en soi une nouveauté. Nous voudrions ici marquer seulement un point qui concerne notre recherche. Reconnaissons d'abord ce qui n'est pas niable : l'importance, dans l'histoire de la philosophie, de la distinction entre l'être et l'étant, et corrélativement entre l'essence et l'existence, et sa présence éclatante dans l'œuvre d'un penseur médiéval, Thomas d'Aquin. Mais si l'on veut en faire une manière de différence spécifique qui caractériserait la philosophie du Moyen Âge chrétien, ou encore un propre qui donc n'appartiendrait singulièrement qu'à elle, on est conduit à des conséquences historiques étranges. Il faut en premier lieu revoir la référence à l'Exode, en notant les diverses façons dont ce texte a été lu. On en trouve les premières interprétations, tout naturellement, dans le judaïsme ancien et médiéval. Or jusque vers le XII siècle le Nom divin révélé à Moïse y est interprété comme signifiant la protection apportée constamment par Dieu à son peuple, son éternité, son incognoscibilité, son ineffabilité, la stabilité de son essence ; c'est seulement à partir de Maïmonide que ce nom signifie l'identité en Dieu de l'essence et de l'existence. Or la problématique de l'essence dans ses rapports avec l'existence est ici reçue d'Avicenne ${ }^{9}$ et c'est la lecture de ce même philosophe qui a d'abord inspiré à Thomas d'Aquin sa métaphysique de l'être, dès le Commentaire des Sentences et le De ente et essentia; sa leçon s'entend encore dans la Somme de théologie ${ }^{10}$. L'instrument philosophique grâce auquel est apparue dans un texte biblique la " vérité sublime " qui est au centre de

8. ID., Constantes philosophiques de l'être, Paris, Vrin, 1983, p. 44. La fameuse expression de " métaphysique de l'Exode " se trouve dans L'Esprit de la philosophie médiévale, op. cit. supra n. 1, p. 50, n. 1. - La question des rapports entre la foi et la raison, qu'on peut aussi considérer comme propre à la philosophie médiévale, serait en fait un mauvais critère. C'est, en effet, un problème religieux non seulement en son principe, puisqu'il suppose une révélation divine adressée à des êtres capables de l'accueillir, mais aussi dans ses premiers développements : ce postulat fournit la norme de la sagesse augustinienne (" Si vous ne croyez pas vous ne comprendrez pas "), et le thème ne devient proprement philosophique que lorsqu'il est soumis à un examen d'ordre épistémologique et noétique, c'est-à-dire pas avant le XIII siècle.

9. Georges VAJDA, « Brefs aperçus sur l'exégèse d'Exode, 3,14 dans la littérature rabbinique et en théologie juive du Moyen Âge ", in Dieu et l'être, Paris, Études augustiniennes, 1978, p. 67-74 ; Esther Starobinski-Safran, « Exode 3,14 dans l'œuvre de Philon d'Alexandrie ", ibid., p. 47-55.

10. Émilie ZUM BRUNN, "La "métaphysique de l'Exode" selon Thomas d'Aquin », op. cit. supra n. 9, p. 245-269. 
la pensée de saint Thomas " a donc été élaboré dans l'islam et transmis ensuite au judaïsme et au christianisme. Avicenne lui-même prolonge des spéculations et controverses amorcées dès le IXe siècle de notre ère par les théologiens musulmans à propos du statut qu'il faut reconnaître aux choses non créées ${ }^{12}$. L'Exode ne révèle pas d'emblée une métaphysique de l'exister : on l'y trouve quand on l'a déjà. Reste que la formation de cette métaphysique, selon ce premier examen, nous laisse bien dans la tranche d'histoire qu'on assigne classiquement au Moyen Âge, même si l'on doit admettre un décalage géographique, puisqu'il faut la situer au IX siècle en Mésopotamie musulmane.

Toutefois les choses sont moins simples, car le thème de l'antériorité de l'être sur l'étant a été formulé bien plus tôt, dans un commentaire du Parménide publié et étudié par P. Hadot, qui l'attribue avec de bonnes raisons à Porphyre. Le parallélisme postulé par les néoplatoniciens entre les deux premières hypothèses de ce dialogue de Platon et les deux premières hypostases de leur système : l'Un-Bien et l'Un-Étant, induit le commentateur à voir dans le premier l'einai, l'être, dont participe l'on, l'étant, qui est le second. Donc l' " identification entre Dieu et l'êtreinfinitif ", comme dit P. Hadot, n'est pas une idée étrangère à la philosophie païenne. Sans doute ce commentaire n'a guère laissé de traces dans les spéculations ultérieures, sinon chez Marius Victorinus, mais cela n'a pas été plus loin ; mais, et c'est le plus important, " cette doctrine se situe très clairement dans la logique du platonisme » et de son principe de participation ${ }^{13}$. Concluons d'abord que la philosophie de l'esse a pris au moins deux chemins (et même si l'un d'eux n'a apparemment « mené nulle part ") avant que les médiévistes d'Occident ne la voient apparấtre chez Thomas d'Aquin. Ajoutons que si l'on persiste à en faire la caractéristique essentielle de la pensée médiévale, celle-ci sera donc née deux fois, et d'abord aux premiers temps du néoplatonisme. Notons ce point, il nous deviendra familier. Notons, en outre, que le critère du contenu est peu sûr : tantôt il nous conduisait à poser des barrières infranchissa-

11. Ainsi s'intitule le chap. 4 de la Première Partie du Thomisme d'É. Gilson, op. cit. supra n. 6 : « Haec sublimis veritas »; la formule est tirée de la Summa contra Gentiles, I, 22 : Hanc autem sublimem veritatem Moyses a Domino est edoctus, "cette vérité sublime, Moïse l'a apprise du Seigneur »; voir ibid., p. 136.

12. J. Joliver, « Aux origines de l'ontologie d'Ibn Sinä », in Études sur Avicenne, dir. par Jean Jolivet et Roshdi RASHED, Paris, Les Belles Lettres, 1984, p. 11-28.

13. Sur tout cela voir Pierre HADOT, Porphyre et Victorinus, Paris, Etudes augustiniennes, 1968 ; et du même, « Dieu comme acte d'être dans le néoplatonisme. À propos des théories d'É. Gilson sur la métaphysique de l'Exode ", in Dieu et l'être, op. cit. supra n. 9, p. 57-63 (Boèce y est désigné, p. 63, comme ayant transmis du néoplatonisme au Moyen Âge la distinction de l'être et de l'étant : le point est à noter, et à retenir quand nous retrouverons ce philosophe). 
bles entre les sphères et les époques, maintenant il les fait refluer l'une dans l'autre. Tentons donc une autre voie, celle que jalonnent les figures des philosophes.

\section{La longue survie de la philosophie antique.}

Les Médiévaux ont eu généralement une vue bien découpée de l'histoire doctrinale ; la répartition chronologique s'y double d'une différence dans l'autorité reconnue aux écrits des diverses époques. En science sacrée, et indépendamment de l'Écriture et des documents élaborés par l'Église (documents conciliaires, textes liturgiques), il y a d'abord les docteurs de l'Église, les sancti, puis ceux qui ont travaillé à leur suite - dans des monastères, comme Anselme de Cantorbéry ou Bernard de Clairvaux, ou dans les écoles et universités ; les références aux premiers ont un poids bien supérieur à celles qu'on peut faire aux seconds, eux-mêmes inégaux entre eux. En philosophie, il y a les auctores, tels Aristote, Cicéron, Priscien, qui ont fondé les sciences, puis les magistri. Sancti et auctores sont les seuls dont les écrits fassent autorité ; leurs successeurs les commentent, les citent à l'appui de leurs propres thèses ${ }^{14}$. Nous avons donc ici deux couples d'ensembles bien distincts, qu'il n'est pas possible de mêler les uns aux autres : Abélard et Porphyre en philosophie, Hugues de SaintVictor et saint Augustin quand il est question des choses divines, diffèrent par le poids doctrinal comme par l'époque. Toutefois, aux limites de cette double série se détache, seul de son espèce, un personnage qui participe des deux groupes à la fois : c'est Boèce. Pour les Médiévaux, qui l'ont beaucoup glosé, c'est une autorité ; mais lui-même avait projeté de rassembler dans ses écrits le savoir antique, ou du moins la matière entière des arts libéraux, et de traduire en latin et commenter tout Aristote et tout Platon. Il est donc placé à une sorte de ligne de partage, autant quant à la chronologie (il mourut en 524 ou 525) que par sa situation intellectuelle, moyen terme dans deux proportions qui auraient pour extrêmes les penseurs antiques et les Médiévaux, les auteurs et les commentateurs.

Son œuvre, interrompue par une fin prématurée, comporte trois parties : des traductions, commentaires et traités qui concernent la logique, la rhétorique et les arts du quadrivium (les mathématiques donc); une œuvre philosophique originale, la Consolation de philosophie ; et plu-

14. Voir entre autres Marie-Dominique CHENU, La Théologie au XIIr siècle, Paris, Vrin, 1957, notamment les chap. 15 et 16 ; et J. JoLIVET, Arts du langage et théologie chez Abélard, Paris, Vrin, $2^{2}$ éd., 1982, p. 176 et suiv. ; p. 246-247. 
sieurs opuscules théologiques. À parcourir successivement ces diverses œuvres, on perçoit mieux la situation singulière de Boèce dans l'histoire de la pensée, situation qui fait que Martin Grabmann a pu l'appeler « le dernier Romain, le premier scolastique ${ }^{15}$. Donc, Boèce a traduit et commenté l'Organon d'Aristote, l'Introduction (Isagoge) de Porphyre à la logique aristotélicienne; il a commenté les Topiques de Cicéron et écrit des traités personnels sur divers points de logique et de rhétorique. Ses traductions, certaines d'entre elles du moins, ont constitué jusqu'au XII' siècle la seule ouverture sur l'œuvre d'Aristote ; ses commentaires y ont donné un accès meilleur, en tout cas plus large. Mais, en outre, ils ont été eux-mêmes commentés, ainsi que ses traités. On rappellera seulement ici que c'est son commentaire de l'Isagogè qui pose pour la première fois la célèbre question des universaux ${ }^{16}$. Ses monographies sur les syllogismes (les catégoriques et les hypothétiques) et sur les mathématiques seront pendant longtemps les seuls ouvrages de référence pour des domaines du savoir où l'on ne connaissait pas encore les œuvres originales ${ }^{17}$. La Consolation a figuré, note M. Grabmann, dans toutes les bibliothèques médiévales ; elle est l'une des quelques voies par lesquelles le platonisme a été connu au Moyen Âge, et au moins l'un des poèmes qui y figurent a été abondamment commenté ${ }^{18}$. Quant aux quelques opuscules théologiques, ils ont été médités et commentés dès l'époque carolingienne (Alcuin, Hincmar de Reims, Jean Scot, Rémi d'Auxerre) et plus tard par Thierry de Chartres, Gilbert de la Porrée, Clarembaud

15. Martin GrabmanN, Die Geschichte der scholastischen Methode, t. I, Freiburg i. B., 1909, 3e partie ; voir aussi toutes les histoires de la philosophie médiévale, et notamment pour le point qui nous intéresse ici, H. LIEBESCHütz, "Boethius and the Legacy of Antiquity ", in The Cambridge History of Later Greek and Early Medieval Philosophy, ed. by A. H. ARMSTRONG, Cambridge, Cambridge University Press, 1967, chap. 35 ; en outre M.-D. ChenU, op. cit. supra n. 14, chap. 6 (" Aetas Boetiana "). Remarquons qu'Edward Kennard RAND, qui appelle simplement Boèce " le premier des scolastiques ", manque en cela un trait capital de son sujet : cf. Founders of the Middle Ages, 1928 ; rééd. New York, 1957, chap. 5.

16. Telle qu'elle est posée par Boèce, l'alternative est la suivante : genres et espèces sont-ils des choses, ou des idées ? Les Médiévaux la transformeront d'une manière significative pour l'histoire de la philosophie : sont-ils des choses ou des mots ? Voir plus loin.

17. Boèce avait traduit tout l'Organon, mais la majeure partie en était devenue à peu près inaccessible.

18. La Consolation fait alterner les développements ou dialogues en prose, les élévations et commentaires lyriques en vers. Le fort beau poème auquel on fait allusion ici est le neuvième du Livre III ( $O$ qui perpetua mundum ratione gubernas...). Voir là-dessus, et pour la bibliographie, Edouard JEAUNEAU, « Un commentaire inédit sur le chant $O$ qui perpetua de Boèce ", Rivista critica di storia della filosofia, 14, 1959, p. 60-80 ; repris in Lectio philosophorum, Amsterdam, Adolf M. Hakkert, 1973, p. 309 et suiv. Quant à la diffusion de la Consolation au Moyen Âge, $H$. LieBeschüTz parle de « centaines de manuscrits " du vilJc au Xve siècle, in op. cit. supra n. 15, p. 538. 
d'Ar s, Thomas d'Aquin ${ }^{19}$. M. Grabmann fait observer que dans bea' coup de ces ouvrages apparaissent pour la première fois des mots ou expressions qui seront repris par les Médiévaux ${ }^{20}$. Ainsi, conclut-il, " le dernier Romain » a donné le premier l'exemple d'une application de la philosophie aux dogmes du christianisme et fondé dans ses opuscules « les éléments de la méthode scolastique " ${ }^{21}$. Ajoutons qu'il a donné aussi le modèle du commentaire de texte, genre si répandu au Moyen Âge. Mais, précisément, nous retrouvons ici un point déjà noté, car ce genre avait été d'abord élaboré dans les écoles philosophiques païennes. Une histoire de la méthode scolastique peut insister à bon droit sur ce qui chez Boèce est l'amorce de développements futurs ; mais lui-même, on l'a dit, voulait, au profit de son temps ${ }^{22}$, récapituler le passé.

Ce caractère ambigu de la pensée de Boèce se manifeste dans le contraste entre les opuscules théologiques et la Consolation, où le lecteur ne trouve rien qui tranche, quant au contenu, avec la philosophie antique : stoïcienne au début, platonicienne ensuite, cette œuvre propose une sagesse tournée vers le divin mais ne fait aucune allusion à la foi chrétienne. C'est une religion philosophique qui pourrait sans doute laisser place au christianisme : or cette place reste vide. On s'est longtemps demandé si le Boèce théologien était le même que le Boèce philosophe, et si ce dernier n'avait pas révélé dans cette Consolation écrite en prison, tout près de la mort, sa vérité spirituelle cachée jusque-là sous un christianisme purement politique. Il y a à peine un siècle que la découverte d'un fragment de Cassiodore a ramené cette œuvre et cette figure à l'unité ; que celle-ci ait pu être mise en doute avec de bonnes raisons fait mesurer combien Boèce était imprégné de la philosophie antique, c'està-dire païenne ; il l'était au point de l'avoir fondue, dans son intimité

19. Outre M. GrabmanN, op. cit. supra n. 15, p. 166-169, qui fait mention d'un certain nombre de manuscrits, voir Gangolf ScHRIMPF, Die Axiomenschrift des Boethius (De Hebdomadibus) als philosophisches Lehrbuch des Mittelalters, Leiden, E. J. Brill, 1966.

20. Op. cit. supra n. 15, p. 157 , à propos du vocabulaire logique; p. 160 et 175 , à propos des définitions formulées dans la Consolation (beatitudo, I. III, pr. 2 ; providentia, l. IV, pr. 6 ; fatum, ibid. ; aeternitas, l. V, pr. 6), et dans les opuscules théologiques (persona, dans le Contra Euticen et Nestorium); et p. 160, à propos de principes qui seront admis comme évidents ; p. ex., omnis cognitio est per modum cognoscentis (Consolation, 1. V, pr. 4).

21. Ibid., p. 169 ; p. 175-176.

22. C'est un point sur lequel insiste H. LIEBeschüTz, op. cit. supra n. 15 , p. 542-543; 546-547. On saisira mieux le sens historique de l'œuvre de Boèce si on la compare à une autre de celles qui ont formé la pensée médiévale : celle de saint Augustin, tellement plus vaste et plus profonde. Boèce a laissé au Moyen Âge des modèles, des formules, et un exemple prestigieux mais point trop écrasant. Augustin a laissé un nombre considérable d'idées, de schèmes, de pistes à suivre, voire de difficultés (en soi, et aussi quand il a fallu, par exemple, concilier deux autorités incontournables : la sienne et celle d'Aristote) ; mais si chacun ou presque s'en est inspiré, personne n'a réellement marché sur ses traces. 
personnelle, à sa croyance religieuse. Dans une étude minutieuse, P. Courcelle a montré combien la Consolation était redevable à Proclus, et avec quelle quasi-certitude on pouvait y déceler l'influence d'Ammonius. Celleci se manifeste plus nettement encore dans le programme de traductions et de commentaires que Boèce s'était tracé, et dans la façon techniquement très précise dont sont rédigés les prolégomènes aux commentaires qu'il a effectivement composés. Ammonius, qui, après avoir été l'élève de Proclus, a été le maître de Damascius, Simplicius, Jean Philopon (notamment), a commencé d'enseigner à Alexandrie en 485 : or, si l'on n'a pas de preuves explicites que Boèce y ait étudié, un certain nombre de faits de la vie de son père en laissent intacte la possibilité, voire en suggèrent la vraisemblance. Il reste vrai de toute façon que Boèce a transmis à l'Occident " la pensée des commentateurs alexandrins les plus récents ", de quelque façon dont il en ait eu connaissance ${ }^{23}$. Ainsi donc il semble être à la fois païen et chrétien, d'Alexandrie et d'Italie, philosophe à l'antique et auteur médité par saint Thomas - en somme, pour en revenir à la formule de Grabmann, le dernier Romain et le premier scolastique. On a parlé plus haut d'une frontière où il se tiendrait, tourné à la fois vers deux mondes. Mais ce prétendu Janus pourrait bien n'avoir qu'un visage ; il ne serait qu'un moment dans une continuité que nous cacheraient de simples habitudes intellectuelles ; continuité complexe peutêtre, mais dont il nous faut examiner de plus près la nature. L'examen de l'œuvre de Boèce tel qu'on vient de le faire, à la suite notamment de Grabmann, reste en effet trop abstrait pour qu'on puisse s'en contenter. Nous devons maintenant aller plus au fond, c'est-à-dire tâcher de saisir dans les formes littéraires, des tendances, des motivations, des tours d'esprit - en un mot les éléments d'un régime mental dont on verra en même temps s'il persiste ou non de l'Antiquité, du moins la tardive, jusque dans le Moyen Âge.

La vie intellectuelle de cette dernière époque est, on l'a dit, largement tributaire de textes : textes sacrés, textes profanes; d'où l'abondance des commentaires et la diversité de leurs formes. Les plus simples, ceux qui répondent aux besoins les plus élémentaires d'une culture qui renaît, ou d'étudiants à leurs débuts, consistent en des gloses littérales qui expliquent le sens des mots rares ou ambigus, en y ajoutant éventuellement des explications plus détaillées, ou portant sur un ensemble plus large que le mot ou la phrase : toutes les variétés sont possibles. La forme canonique d'une glose complète (expositio) est donnée au XII siècle par

23. Pierre Courcelle, « Boèce et l'école d'Alexandrie ", Mélanges d'archéologie et d'histoire de l'École de Rome, 52, 1935, p. 185-223 ; ID., Les Lettres grecques en Occident de Macrobe à Cassiodore, Paris, E. de Boccard, 1943, p. 298-300. 
Hugues de Saint-Victor qui distingue trois niveaux dans le texte et sa compréhension : la lettre (littera), qui demande une explication grammaticale ; la signification (sensus), c'est-à-dire la teneur de la phrase ; la pensée (sententia), qui réclame une interprétation de l'ensemble du passage et la perception de la doctrine qu'y a mise l'auteur ${ }^{24}$. C'est bien selon ce schéma que s'organisent tous les commentaires, privilégiant sans doute l'une ou l'autre des rubriques, se compliquant de perspectives autres s'ils portent sur des écrits révélés - mais finalement tout peut se ranger sous les trois chefs qu'on a dits. La tendance à commenter est si profonde dans l'esprit des intellectuels médiévaux qu'on peut la reconnaître sous des formes apparemment éloignées de celle des commentaires traditionnels : je veux dire dans les Sommes de théologie. Celles-ci, en effet, ne font que reprendre, sous une autre présentation, les commentaires du Livre des sentences qu'avait compilés Pierre Lombard vers le milieu du XII siècle ; or ce travail consistait en un rassemblement, selon un plan systématique, des opinions soutenues par des auteurs de poids sur les diverses parties de la croyance chrétienne. Commenter ce livre, c'est examiner ces opinions, notamment dans la mesure où elles divergent ou soulèvent des difficultés, et proposer une solution à chacun de ces divers problèmes. Cet examen ne peut être fait au hasard : il doit se dérouler selon des procédures dialectiques bien réglées ; ainsi le Livre et le commentaire se déploient en une diversité de questions dont chacune traite d'un point particulier ; ces questions se subdivisent elles-mêmes en articles. Ainsi se constituent la matière et la forme des Sommes : ces monuments dogmatiques ont leur germe dans la lecture des textes et leur commentaire. Or ce genre de commentaire par questions n'est pas réservé à la théologie : citons à titre d'exemples les Questions sur Priscien de Radulphus Brito, les Questions sur le Livre des causes de Siger de Brabant... ; le texte de l'auteur y est suivi de plus ou moins loin (ce ne sont pas des gloses), mais c'est bien lui qui guide la réflexion du commentateur ${ }^{25}$. Il est évident que ces formes sont typiquement médiévales ; celle de la question, qui en est comme la cellule mère, résulte des conditions effectives de l'enseignement et de l'importance prise, corrélativement, par l'exercice de la dialectique et la pratique de la grammaire : or précisément nous reviendrons sur ce point. Sans modèle antique donc, et d'autant moins qu'avant d'atteindre au XIII' siècle sa forme classique elle s'était lentement élaborée, elle fait écho cependant aux exposés doxographiques et aux discussions dont Aristote fait précéder les exposés de ses vues per-

24. Didascalicon, PL 176, 771 D.

25. Voir pour plus de détails M.-D. CHENU, Introduction à l'étude de saint Thomas $d^{\prime}$ Aquin, Paris, Vrin, $2^{`}$ éd., 1954, p. 66-83 et 255-258, notamment. 
sonnelles. Elle est comme une résurgence spontanée de cette méthode, mais transformée et appliquée au commentaire. Quant à ce dernier genre, au sens simple et strict du mot, nous avons déjà vu que Boèce en avait donné le modèle, emprunté d'Alexandrie. Ainsi les commentaires grecs des œuvres d'Aristote et de Platon ne constituent qu'une part de la littérature en ce domaine : il conviendrait, pour là rassembler en la diversité de ses contenus, d'ajouter à ces longues séries tous ceux au moins qu'ont produits les successeurs de Boèce ; d'y joindre aussi, comme représentants d'un même genre, les commentaires médiévaux sur d'autres auteurs antiques. Cette manière de pratiquer la philosophie et donc de la concevoir assure sur ce point une continuité sans faille entre l'Antiquité finissante et le Moyen Âge ${ }^{26}$.

On peut ranger beaucoup moins d'œuvres dans la suite de la Consolation que dans celle des commentaires : pour leur part la plus grande, et de beaucoup, les spéculations des Médiévaux restent dans l'orbe de la foi chrétienne. On peut toutefois repérer, au long du Moyen Âge, des témoins d'une pensée philosophique dégagée de ce présupposé. Ce sont pour la plupart ces philosophes qu'on appelle " averrö̈stes ", d'un nom souvent impropre mais commode parce que traditionnel. Le nom de « philosophes ", précisément, leur conviendrait beaucoup mieux ${ }^{27}:$ les premiers de leur lignée sont des maîtres ès arts qui, chargés du fait de leur fonction d'expliquer Aristote, s'en tiennent par méthode à la pure philosophie, sans tenir compte dans leur exégèse, ni bien entendu de ce que l'Église enseignait par ailleurs, ni même des conséquences que pourrait avoir, par rapport à l'enseignement de la théologie confié à une autre faculté, une interprétation purement immanente des textes inscrits à leur programme. Le nom d'averroïstes leur a d'abord été attribué parce qu'ils adoptaient les vues d'Averroès sur l'obscure doctrine aristotélicienne de l'intellect (on se demande notamment s'il est unique pour l'espèce humaine

26. "Pendant près de deux mille ans la philosophie a été conçue comme exégèse ", écrit Luc BrIsson, in Porphyre, la vie de Plotin, Paris, Vrin, 1982, p. 57, qui renvoie à P. HADOT, "Philosophie, exégèse et contre-sens ", Actes du XIVe Congrès international de philosophie (1968), I, Vienne, 1970, p. 333-339.

27. Fernand Van Steenberghen, La Philosophie au xIme siècle, Louvain/Paris, Publications universitaires/Béatrice Nauwelaerts, 1966, discute la formule - et l'idée - d' « averroïsme latin " mise en circulation par Renan (voir notamment p. 364 et suiv., et, à propos de Siger de Brabant plus particulièrement, p. 399-400). Il propose la formule d' " averroïsme hétérodoxe ", " désignation brève, mais exacte ", dit-il, de ce mouvement à ses débuts. Je pense que le meilleur nom à donner à ses représentants est celui de " philosophes ", par contraste avec les théologiens ; ce nom a en outre l'avantage de convenir aussi à tous leurs successeurs, fussent-ils alexandristes : ils travaillent tous en continuité avec les philosophes antiques, laissant de côté dans l'élaboration de leurs doctrines ce fait religieux, c'est-à-dire extra-philosophique en son origine, qu'est le christianisme. 
entière, ou si chaque homme a le sien propre ; il est unique, dit Averroès). Mais ce sont principalement des philosophes aristotéliciens, et ce double caractère fonde seul l'unité du courant dit averroïste, qui dans toutes les universités traverse la seconde moitié du Moyen Âge et se prolonge jusqu'au XVII siècle ; Averroès n'est qu'une de leurs sources, Alexandre en est, pour certains d'entre eux, une autre ; on en voit même se placer assez près d'Augustin. L'essentiel en tout cela est que, dans leurs écrits, ils ne font nulle référence au christianisme, sauf à l'occasion quelques clauses dont on ne peut savoir si elles sont de pure forme ou non. Tels sont les textes ; savoir ce que leurs auteurs croyaient ou non est sans importance, à la différence de Boèce. Ils lui ressemblent en ce que leur morale est une morale de philosophes, purement humaine, définissant la béatitude et les vertus en conformité avec l'Éthique à Nicomaque et non avec l'Évangile. Ce courant est né et s'est développé, on l'a dit, du fait de l'organisation des études dans l'Université. Mais, déjà au XII' siècle, on peut leur trouver deux précurseurs - non en aristotélisme bien entendu, mais pour cette attitude purement philosophique. Leurs noms ont été cités plus haut. Le De eodem et diverso d'Adélard de Bath commence par un dialogue entre deux figures féminines : Philosophie (comme chez Boèce), l'amie de la sagesse, et Philocosmie, l'amie du monde ; chacune à son tour fait valoir à Adélard les biens qu'elle pourra lui apporter ; c'est évidemment Philosophie qui l'emporte, avec ses suivantes qui sont les disciplines libérales - et rien qu'elles. Le Dieu de Bernard Silvestre, celui en tout cas qu'il évoque dans sa Cosmographia, peut bien à un moment être décrit comme un triple rayon réfléchi sur soi-même (on pense alors à la Trinité chrétienne) : il reste très loin du monde, sa Sagesse est, non un Fils qui s'incarne, mais une Fille, Noys, chargée d'organiser une matière (Hylè) dont il n'est pas dit qu'elle ne soit pas éternelle. Les quelques allusions scripturaires semées dans la longue suite de vers qui retrace l'histoire du monde sont loin de contrebalancer la teneur profane de ce texte, qui puise à toutes les sources antiques accessibles à l'époque. Entre ces philosophes qu'on vient d'évoquer brièvement, et ceux de l'Antiquité, il n'y a pas de discontinuité foncière, ils en sont les héritiers authentiques en plein cœur du Moyen Âge.

Les opuscules théologiques constituent le troisième côté de l'œuvre de Boèce. Tout naturellement Grabmann ne s'y intéresse que dans la mesure où ils suivent déjà une méthode qui sera l'une des caractéristiques principales de la scolastique : l'application de la philosophie (celle d'Aristote, dit-il, ce qui dans le cas de Boèce est inexact) au contenu de la foi chrétienne. Mais si ces petits traités sont remarquables à cause de leurs suites, on peut aussi les rapprocher de leurs antécédents païens. Pour étudier la Trinité, la personne et les deux natures du Christ, Boèce dispose 
d'un fond dogmatique constitué par l'Écriture telle que l'avait interprétée la tradition catholique affirmée et cristallisée dans les conciles, notamment ceux de Nicée et de Chalcédoine. Ce philosophe chrétien dont on a vu plus haut qu'il était néoplatonicien, fait travailler sur un objet religieux sa pensée nourrie de la tradition antique, et il en résulte de l'un à l'autre un apport réciproque de clarté et de précision. Or ce n'était pas là une chose nouvelle : dans le paganisme même des philosophes avaient accepté des révélations et en avaient fait l'objet d'une réflexion philosophique. Ainsi Porphyre avait tiré de divers oracles, et exposé de façon systématique, des enseignements concernant, entre autres choses, la piété, le respect dû aux dieux, les règles de leurs mœurs (politeia), le destin ${ }^{28}$. Il y attache autant de foi qu'un chrétien à ses livres sacrés : « il est sûr de lui et inébranlable, celui qui puise à cette source [...] ses espérances de salut $"{ }^{29}$; il expose sa méthode et ce qu'il en attend avec une précision parfaite : «le présent recueil comportera l'exposition d'un grand nombre de principes philosophiques contenant la vérité que les dieux ont voulu exprimer dans leurs oracles $"{ }^{30}$. Certes, cette Philosophie est une œuvre de jeunesse, et plus tard Porphyre, dans sa Lettre à Anebo, refusera les rites magiques et théurgiques qu'il avait d'abord admis et critiquera les cultes mystériques. Mais dans les Mystères d'Égypte, écrits pour réfuter la Lettre à Anebo, Jamblique prend la défense de ces pratiques et, ce qui nous intéresse surtout, cite des témoins prestigieux de cette attention révérentielle prêtée aux oracles : « Pythagore, Platon, Démocrite, Eudoxe et bien d'autres parmi les anciens Grecs ont trouvé l'enseignement convenable dans les inscriptions sacrées de leurs temples $"{ }^{31}$. Il y a pour lui, entre la philosophie et les antiques sagesses révélées, une identité foncière qui lui permet par exemple d'interpréter des traditions sacrées égyptiennes comme si elles signifiaient, sous les noms de trois dieux, l'Un

28. Cette Philosophie tirée des oracles est perdue ; on en a toutefois un certain nombre d'extraits cités par divers écrivains. Voir Gustav Georg WolfF, Porphyrii de Philosophia ex oraculis haurienda librorum reliquiae, Berlin, 1856 (réimpression reprographique, Hildesheim, Olm, 1962).

29. Cité par EuSĖBE DE CÉSARÉ, La Préparation évangélique, livres IV-V, I-17, introd., trad. et annotation par Odile Zink, texte grec rév. par Edouard DES PLACES, Paris, Cerf ("Sources chrétiennes ", 262), 1979, p. 121 (= IV, 7, 1).

30. Ibid. ( = IV , 7, 2); cf. G. WolfF, op. cit. supra n. 28 , p. 38 : « philosophia ex oraculis explicatur. " Dans cet ouvrage, il est aussi question des rites, mais cela ne nous concerne pas.

31. Jamblique, Des mystères d'Égypte, texte ét. et trad. par É. Des Places, Paris, Les Belles Lettres, 1966, p. 39 (2-3 Parthey). Il ajoute à l'adresse de Porphyre : " toi, notre contemporain, animé du même esprit que ces grands hommes "; cela semble aller au-delà de la simple politesse : malgré leur désaccord, il ne considère pas que Porphyre ait entièrement renié ses premières vues. 
indivis, l'Intellect qui se pense lui-même, et l'Intellect démiurgique ${ }^{32}$. Il est le premier à avoir fait des Oracles chaldaïques la « bible du néoplatonisme » (selon l'expression de P. Hadot), que scruteront d'autres philosophes : tel Proclus, qui pense que ces oracles contiennent un système identique à ceux de Platon et des traditions orphiques ${ }^{33}$. Ainsi Boèce n'est que l'un des nombreux philosophes néoplatoniciens qui se sont comportés en théologiens en se fondant sur des révélations divines. Certes, la différence entre le contenu de ces oracles et celui du Nouveau Testament, interprété en outre par une tradition et des conciles, est très importante du point de vue religieux ; elle n'est pas pertinente si l'on ne considère que la méthode qu'on a dite et ses présupposés, selon lesquels il existe, outre la spéculation rationnelle et antérieure à elle, une source de savoir dont l'origine est au-dehors et au-dessus de l'esprit des hommes. Ici encore Boèce, qu'on ne peut que placer dans ce courant, assure la continuité entre l'Antiquité tardive et le Moyen Âge.

Mais aux genres et aux méthodes qu'il a pratiqués et transmis aux Médiévaux on peut en joindre d'autres qui étaient familiers aux derniers philosophes païens et qu'on retrouve, à peine transposés, dans les siècles qui suivent. Les œuvres composées selons ces normes sont, dans tous ces cas, " étroitement liées à des conduites orales », comme le note $\mathrm{P}$. Hadot dans une étude consacrée à la philosophie antique ${ }^{34}$. Ce sont par exemple les diatribes, qui traitent d'une question donnée en remontant aux principes de sa solution et argumentent dans ces limites précises ; on peut rattacher à ce genre des ouvrages comme ceux de Cicéron, Plutarque, Sénèque, Plotin ${ }^{35}-$ mais aussi, ajouterons-nous, toute une part de la litté-

32. Ibid., p. 196 (262-263 Parthey); avant eux se place une première triade, antérieure aux " êtres véritables " et aux principes. Sur les difficultés posées par les noms cités dans le texte, voir la note, ibid., p. 220-221.

33. Voir Hans LEwy, Chaldean Oracles and Theurgy, nouv. éd. par Michel TARDIEU, Paris, Études augustiniennes, 1978 , p. 481-485 ; notamment, p. 483-484, un tableau synoptique des trois systèmes, selon Proclus. - L'expression de $P$. Hadot citée ci-dessus est dans Porphyre et Victorinus, op. cit. supra n. 13, p. 26. - Sur cet aspect de la philosophie antique voir l'introduction d'Henri-Dominique SAFFrey à Proclus, Théologie platonicienne, I, Paris, Les Belles Lettres, 1968 : l'école d'Athènes « essayant d'épanouir la fleur du platonisme au soleil mystique de la religion païenne is ( $p$. xxxvi) ; les commentaires des Oracles chaldaïques composés par Porphyre, Jamblique, Proclus (passim). On notera au passage le programme de l'Académie au moment où Proclus y étudiait : Aristote, Platon, puis les textes théologiques : poèmes orphiques, Oracles chaldaïques (p. xv-xvi) ; cela évoque par avance celui d'une université médiévale, quant à l'ordre et l'esprit de cette série. Voir le travail récent de $\mathrm{P}$. HADOr, " Théologie, exégèse, révélation, écriture, dans la philosophie grecque ", in Les Règles de l'interprétation, M. TARDIEU, éd., Paris, Cerf, 1987, p. 13-34.

34. Leçon inaugurale au Collège de France (18 février 1983), p. 28. Voir aussi, du même, Exercices spirituels et philosophie antique, Paris, Études augustiniennes, 1981, chap. 2, « Exercices spirituels antiques et philosophie chrétienne ».

35. Ibid., p. 31. 
rature monastique. Dans le cloître comme dans l'école, l'abbé comme le maître a devant lui des auditeurs dont il a la charge spirituelle ; ce sont des entretiens ou des dialogues tenus en ces circonstances - ou imaginés - que transmettent les ouvrages de ce type : la collatio monastique est, par son origine et sa structure, l'héritière de la diatribe. Dans un genre voisin, il faut ranger les écrits de direction spirituelle : leur caractéristique est de se régler sur le niveau spirituel de la personne ou du groupe à qui il est destiné ; ils sont composés « non pas tant pour informer le lecteur au sujet d'un contenu doctrinal, que pour le former, en lui faisant parcourir un certain itinéraire au cours duquel il va progresser spirituellement ${ }^{36}$. À cette intention répondent les collections de sermons : que leur suite soit fixée par le calendrier liturgique ou par le plan d'un texte sacré qu'ils commentent (ainsi des sermons de saint Bernard sur le Cantique des cantiques), elle doit déterminer chez les auditeurs, puis les lecteurs, un progrès scandé par la succession des célébrations ou l'économie du livre révélé. On peut en rapprocher les écrits qui condensent en quelques pages la description du chemin que l'âme doit parcourir pour arriver à l'extase : tel l'Itinerarium mentis in Deum de saint Bonaventure ${ }^{37}$.

Arrivés à ce point, nous voyons que, du moins pour la forme et l'intention de leurs écrits, rien ne distingue radicalement les philosophes du Moyen Âge, ni même ses théologiens, de ceux de l'Antiquité, particulièrement en ses derniers siècles. Les uns et les autres ont les mêmes objets : remplir leurs devoirs de guides spirituels ; expliquer les écrits des philosophes du passé ; commenter les écrits révélés pour en tirer des instructions sur les réalités divines et sur la conduite qu'il faut tenir à l'égard

36. Ibid., p. 33. L'auteur cite encore parmi ces genres « la lecture et l'exégèse des textes faisant autorité » (p. 31-33) : mais on a déjà parlé des commentaires. Il note aussi, p. 34, la nécessité pour la philosophie antique de reprendre des formules traditionnelles, quitte à leur donner un sens qu'elles n'avaient pas à l'origine ; c'est là aussi un procédé bien connu des Médiévaux, obligés de se référer à des " autorités " qui, isolées de leur contexte, se laissaient manier presque à volonté ; c'est cette facilité qui faisait dire à Alain de Lille que l'autorité avait " un nez de cire, ployable en tous sens ", et, plus brutalement, à Adélard de Bath, que c'était « une putain ".

37. Bien entendu les religieux qui, au Moyen Âge, prononçaient des sermons ou tenaient des collationes réglaient leur pensée et leur expression sur les Pères bien plus que sur Cicéron et Sénèque (que cependant ils n'ignoraient ni ne dédaignaient ; voir sur ce point les travaux de Dom Jean Leclercq). Mais les Pères avaient eux-mêmes conçu leurs écrits selon des modèles littéraires et philosophiques contemporains pour eux, antiques pour nous, païens en tous cas ; cet usage de formes grecques remonte, avant même les Pères, aux tout premiers écrits chrétiens : voir Werner Wilhelm JAEGER, Early Christianity and Greek Paideia, Cambridge Mass./London, Belknap Press of Harvard University Press/Oxford University Press, 1962, p. 7 et suiv. 
des êtres supérieurs. Ces différentes fonctions qu'ils devaient assumer ont donné lieu tout naturellement à certaines formes d'enseignement et donc à certains genres littéraires, dont Boèce, placé historiquement à la lisière de deux mondes - le païen et le chrétien - et de deux époques, a transmis les principaux ${ }^{38}$. Ainsi ont été ouvertes les voies que la spéculation chrétienne a suivies par la suite : celles-là mêmes que lui proposait le monde où le christianisme était né.

Cela ne date évidemment pas du temps de Boèce, que, rappelons-le, un demi-millénaire sépare du temps de Jésus : les tout premiers écrits chrétiens se sont renvoyé la même image d'un christianisme philosophique sophes païens ${ }^{39}$. L'analyse des idéaux grecs et de ceux du christianisme révèle des affinités qui expliquent la facilité avec laquelle ils se sont joints ${ }^{40}$, avant que sur ces bases ne se constitue une philosophie chrétienne qui, du II' au IV ${ }^{e}$ siècle, s'est intégré une part de plus en plus grande de la culture de cette époque ${ }^{41}$. Mais il y avait déjà longtemps que les Juifs (dès la période hellénistique), puis les chrétiens, étaient apparus aux yeux des Grecs comme des philosophes ${ }^{42}$. Ainsi, depuis des temps qui remontent assez loin dans l'Antiquité, philosophes grecs et penseurs chrétiens se sont renvoyé la même image d'un christianisme philosophique en son essence et qui se donne progressivement tous les caractères d'une philosophie entièrement déployée en reprenant à son compte la substance conceptuelle élaborée dans les écoles grecques. L'arrivée des « Barbares " n'interrompt pas le cours de la philosophie antique, il l'appauvrit et le fragmente seulement : Boèce se propose d'en préserver et d'en transmettre le principal (Platon, Aristote, les arts libéraux), et c'est bien cela qui, forme et contenu, passe au Moyen Âge. Malgré le cours tourmenté de l'histoire, le fléchissement de niveau observé dans les siècles qui suivent la chute de Rome, c'est bien la même philosophie qui, en Occident

38. Il est piquant de noter que Boèce n'était lui-même rien de ce qu'on a dit plus haut : ni directeur de conscience, ni professeur de philosophie, ni évêque, c'était un homme de gouvernement ; singularité de plus dans cette curieuse figure.

39. Voir W. JAEGER, op. cit. supra n. 37, ibid. Il ne faudrait pas imaginer que les premiers penseurs chrétiens aient tous accepté la philosophie grecque : certains l'ont rejetée (Tatien, Tertullien), mais dans l'ensemble elle a été assimilée.

40. Ibid., p. 39 et suiv.

41. Ibid., p. 46-47 : comment Clément d'Alexandrie et Origène inaugurent une « philosophie chrétienne [...] qui consiste exclusivement en ce que [Aristote et les Stoïciens] appelaient théologie »; et p. 75 : les Cappadociens (Grégoire de Nazianze, Basile, Grégoire de Nysse) fondent une culture chrétienne complète en intégrant l'ensemble de l'héritage grec. Voir aussi J. WhitTAKER, « Plutarch, Platonism and Christianity », in Neoplatonism and Early Christian Thought : Essays in Honour of A. H. Armstrong, ed. by H. J. BLuMENTHAL and R. A. MARKUS, Londres, Variorum Publications, 1981, p. 50-63.

42. W. JAEGER, op. cit. supra n. 37, p. 33, cite notamment Galien. 
latin du moins, attend le XII ${ }^{e}$ siècle, " âge boécien " ${ }^{43}$, pour se développer extensivement. Certes, la teneur religieuse n'en est plus la même qu'à Alexandrie ou à Athènes ; mais la différence n'est pas telle que l'ont dit les apologistes les plus radicaux, ou les auteurs monastiques que nous citions en commençant cette étude : d'une période à l'autre, on voit perdurer plusieurs grandes images qui donnent du monde et de l'homme des représentations formellement identiques malgré la rupture religieuse provoquée par l'apparition et l'installation du christianisme. On peut citer les représentations associées d'un univers spirituel ; d'une âme menacée qu'il faut sauver en lui donnant le moyen de s'y rendre, ou plutôt de s'y retrouver : ce thème s'exprimant principalement sous l'aspect d'une sortie et d'un retour ; de la méditation et de l'ascèse auxquelles il faut se livrer pour parvenir à ce résultat ; de la providence par laquelle le divin guide secrètement le mortel. Ces thèmes ne se retrouvent pas chez tous les philosophes, mais du moins chez les néoplatoniciens et déjà chez Platon. Il serait inutile d'entrer dans le détail de chacun ; on se contentera d'évoquer une image dont la récurrence, l'apparition en des endroits divers, ne surprendra pas si l'on remarque qu'elle habite plus ou moins visiblement les représentations qu'on a citées. C'est celle de la chaîne d'or, qui apparaît déjà chez Homère ${ }^{44}$. En voici trois témoins, et d'abord Macrobe :

« du Dieu suprême vient l'Intelligence, de l'Intelligence l'Âme ; l'Âme établit et vivifie tout ce qui vient ensuite ; $[. .$.$] toutes choses se suivent en des$ successions ininterrompues, dégénérant à mesure qu'elles descendent. Du Dieu suprême jusqu'à la dernière lie des choses une unique connexion, nulle part interrompue, s'enlace à soi-même par des liens réciproques : c'est la chaîne d'or dont Homère dit que Dieu a ordonné qu'elle descendît du cièl sur la terre $"{ }^{45}$.

Un néoplatonicien chrétien, Marius Victorinus, avait un peu plus tôt dans le IVe siècle usé de cette image : " la chaîne, c'est Dieu, Jésus,

43. Aetas boetiana : l'expression est de M.-D. Chenu, op. cit. supra n. 15. Ainsi R. A. MARKUS, in op. cit. supra n. 41, p. 204, signale « l'immense fécondité de la tradition platonicienne à l'œuvre dans l'esprit des penseurs chrétiens du second au douzième siècle ".

44. Iliade, VIII, 19. Sur l'origine et l'histoire de cette image, voir : Arthur O. Lovesoy, The Great Chain of Being, Cambridge Mass., 1950, chap. 3 ; L. EdELSTEIN, "The Golden Chain of Homer ", in Studies in Intellectual History Dedicated to Arthur A. Lovejoy, 1953, p. 48-66 ; Pierre Lévêque, Aurea Catena Homeri, Paris, Les Belles Lettres, 1959 (avec en appendice, p. 77-81, l'opuscule de Michel Psellos sur la Chaîne d'or); Jean PÉPIN, Mythe et allégorie. Les origines grecques et les contestations judéo-chrétiennes, Paris, Études augustiniennes, $2^{e}$ éd., 1976, p. 122-123.

45. Commentarii in Somnium Scipionis, I, 25 ; éd. Jacobus W. WILlIS, Leipzig, Teubner, 1963, p. 58. 
l'Esprit, l'Intelligence (Nous), l'Âme, les Anges, puis tous les êtres corporels " ${ }^{46}$. Au XII" siècle, Guillaume de Conches la reprend dans ses Gloses sur le Timée :

" Dieu tira de sa pensée un troisième genre d'âme [cf. Timée, 35 a]. Troisième, dans la chaîne d'or d'Homère, car l'Essence divine est telle qu'elle ne vient de rien, la Sagesse divine vient de Dieu, l'Âme du Monde de l'un et de l'autre, les corps célestes de ces trois, les êtres terrestres des quatre $"{ }^{47}$;

dans ses Gloses sur Macrobe, il l'associe à un épisode biblique :

" Pour signifier cette liaison des choses, Homère dit que Jupiter lança du haut du ciel une chaîne qui descend jusqu'à la terre sans discontinuité ; c'est aussi l'échelle que Jacob vit en songe " ${ }^{48}$.

Plusieurs thèmes se lient dans ces trois textes, d'autres en sortent, ainsi que de leur rapprochement. Tous expriment des médiations : la hiérarchie néoplatonicienne foisonnante chez Proclus se transformera chez Denys en une angélologie ; or l'Ange apparaît aussi chez Marius Victorinus explicitement, et implicitement mais sans aucun doute possible, chez Guillaume ${ }^{49}$. Or les fonctions des anges sont analogues à celles des démons du paganisme : l'image de la chaîne d'or ou de l'échelle se raccorde par eux à l'idée de Providence ${ }^{50}$. Ces représentations religieuses s'expriment aussi philosophiquement par la continuité du métaphysique et du physique, dans les trois textes cités ; et encore par l'illumination de l'esprit du fait d'un être supérieur - image amorcée chez Aristote, abondamment développée par ses successeurs et commentateurs, essentielle aux angélologies des trois religions monothéistes, sous une forme soit purement scripturaire soit philosophiquement élaborée. - Cette analyse pourrait se poursuivre sur d'autres plans, évoquer aussi bien la controverse autour d'Arius que la piété pour les saints intercesseurs ${ }^{51}$.

46. Adversus Arium, I, 15 ; éd. P. Hador, Paris, Cerf (« Sources chrétiennes », 68), 1960 , p. 260.

47. Glosae super Platonem, éd. E. Jeauneau, Paris, Vrin, 1965, p. 149.

48. Ibid., note $c$.

49. «Voilà qu'une échelle était dressée sur la terre et que son sommet atteignait le ciel, et des anges de Dieu y montaient et descendaient ", Genèse, 28, 12 (trad. R. DE VAUX).

50. Platon " estime que les démons [...] sont les serviteurs des dieux, les gardiens des hommes et leurs interprètes quand ceux-ci veulent obtenir quelque chose des dieux ", selon Apulée (De Platone, XII).

51. Voir Peter Brown, Le Culte des saints, trad. Aline Rousselle, Paris, Cerf, 1984, chap. 3. Au milieu des pratiques et représentations propres à la religion nouvelle, l'image de la médiation entre le ciel et la terre reste vivace. 
Il suffira d'avoir évoqué, fût-ce sommairement, une image qui traverse époques, religions, philosophies, théologies, et dont la prégnance et la persistance expriment bien la continuité entre la pensée antique et la médiévale.

Ainsi, bien qu'associée à une religion nouvelle, la philosophie médiévale prend l'exacte suite de la philosophie antique. Plus précisément, elle continue le néoplatonisme de l'Antiquité tardive. Elle en partage les représentations ontologiques et cosmologiques, elle en conserve, du moins quant aux intentions essentielles, les genres littéraires et les méthodes : le cas de Boèce, sa situation, son influence sont à cet égard parfaitement clairs. Il fallait insister là-dessus, car plusieurs faits tendent à masquer cette évidence : ce sont l'apparition et le triomphe du christianisme d'abord ; ensuite et surtout les affrontements entre cette nouvelle religion et les autres - polémiques chrétiennes depuis Paul, et antichrétiennes de la part des philosophes païens ; les persécutions du christianisme, puis son installation à côté du pouvoir ; les écrits des Pères qui critiquent les croyances anciennes pour installer à leur place la nouvelle sagesse - ce dont témoigne la présence dans l'œuvre de saint Augustin d'expressions telles que : " notre philosophie chrétienne », « les philosophes qu'étaient les sages, les prophètes ${ }^{52}$. Certes É. Gilson, qui a relevé ces passages, note que " philosophie " signifie ici « religion"; mais si l'on prend garde à ce qu'enveloppe la notion de sagesse (dont la philosophie est l'amour), et au caractère religieux de la philosophie néoplatonicienne, on ne doutera pas qu'Augustin n'envisage un complet remplacement de celle-ci par le contenu de la foi chrétienne et ses développements théoriques. Toutes ces ruptures donc tendent à faire négliger la continuité réelle qui, indépendamment des frontières religieuses, relie à la pensée antique la pensée médiévale. De ce point de vue celle-ci constitue un moment de celle-là, et on est fondé à compter les penseurs du christianisme dans le même ensemble que ceux des diverses écoles néoplatoniciennes ; à voir dans le Pseudo-Denys un terme intermédiaire dans un continuum qui va de Jamblique à Jean Scot, comme le montre $\mathrm{S}$. Gersh ${ }^{53}$, et même bien au-delà, le corpus dyonisien ayant été une autorité pour tout le Moyen Âge ; et les infléchissements et transformations dus à l'adaptation à la religion chrétienne, du néoplatonisme, n'altèrent pas substantiellement cette philosophie ; ils en constituent un nouvel état.

52. L'Esprit de la philosophie médiévale, op. cit. supra n. 1, p. 413.

53. Stephen GERSH, From Iamblichus to Eriugena, Leiden, E. J. Brill, 1978 ; la même constatation s'impose à la lecture de René RoQuEs, L'Univers dionysien, Paris, Aubier, 1954. 
La métamorphose.

Outre la chose et l'idée surgit en tiers le sens des noms: bien que l'autorité ne le dise pas, ce n'est pourtant pas contraire à la raison.

Pierre Abélard, Gloses sur Porphyre.

Voilà du moins ce qui apparaît à un niveau profond, géologique, de l'histoire spirituelle et doctrinale. Ainsi considérée la philosophie antique s'achève à une époque qui culmine en la nouvelle astronomie et la fondation de l'ordre des Jésuites, la philosophie de la Renaissance unissant diversement, en sa richesse confuse, des éléments religieux, esthétiques et rhétoriques, et préparant l'avènement moderne du sujet dans un univers désacralisé. Toutefois, l'histoire, même doctrinale, ne se réduit pas à ses rythmes les plus profonds, et l'on est amené à une conclusion non contradictoire à la précédente, mais autre, si l'on jette un regard différent sur la pensée médiévale. C'est le regard du bibliothécaire. La philosophie médiévale se distingue entre celles de toutes les époques pour avoir produit des ouvrages dont la structure élémentaire est la question. Nous en avons déjà dit quelque chose, pour en marquer la place dans le genre du commentaire et l'affinité avec les exposés doxographiques d'Aristote. Notre analyse sera maintenant différentielle. En commençant par le plus complexe, les livres composés de questions sont d'abord, nous l'avons dit, des commentaires des Sentences de Pierre Lombard ; des Sommes qui transposent ces commentaires; des exposés systématiques d'une science profane, rattachés volontiers à des ouvrages classiques dans le domaine (ainsi l'ouvrage de Radulphus Brito sur les modes de signifier a pour titre Quaestiones super Priscianum minorem : questions, donc, sur la partie de l'Institutio grammatica de Priscien consacrée à la syntaxe) ; des recueils de questions portant toutes sur un même sujet (questions disputées), ou sur des sujets variés (questions quodlibétales). C'est au XIII' siècle que ces genres apparaissent et se fixent ; en effet, ils naissent dans les universités, résultats des nécessités et des méthodes de l'enseignement qui y était dispensé : commentaires des textes de base, discussions réglées selon des normes institutionnelles ${ }^{54}$. Voici donc une première détermination, sociale, de ces productions typiquement médiéva-

54. Sur ces genres, leur origine et leur environnement scolaire, voir Palémon GloRieux, "L'enseignement au Moyen Âge. Techniques et méthodes en usage à la Faculté de Théologie de Paris, au XIII siècle ", Archives d'histoire doctrinale et littéraire du Moyen Äge, XXXV, 1968, p. 65-186, et particulièrement p. 105-133 ; et The Cambridge History of Later Medieval Philosophy, ed. by Norman Kretzmann, Anthony Kenny, Jan Pinborg, Londres/New York/Melbourne, Cambridge University Press, 1982, p. 11-33. 
les. Mais l'élément fondamental qu'est la question remonte plus haut, au début du XII' siècle ; elle naît pour sa part des difficultés rencontrées dans le cours des explications de textes, et se constitue en procédures précises dans les écoles théologiques ; quelques problèmes de détail que pose son analyse ${ }^{55}$, sa technique propre - relevé des apories, analyse sémantique, répartition des matières - implique une pratique développée des arts du langage, grammaire, rhétorique, dialectique : c'est bien pourquoi le premier grand logicien du Moyen Âge donne dans son Sic et non un long relevé de textes bibliques et patristiques qui paraissent contradictoires quand on les rapproche, et une méthode historique et linguistique pour résoudre ces difficultés. Cette œuvre d'Abélard n'est pas radicalement innovatrice, mais elle fait passer à l'état réfléchi et au plan de la méthodologie une pratique déjà courante. Or nous avons ainsi rencontré le second principe de la question : le principe scientifique, après l'institutionnel, et du même coup le véritable élément différentiel de la pensée médiévale, ce qui constitue sa vraie nouveauté par rapport à l'antique, plus décisive que les modifications du néoplatonisme : l'importance majeure prise en elle par le langage et les disciplines qui en traitent, et leurs applications à la théologie et à la philosophie.

Pendant longtemps les études de philosophie médiévale se sont concentrées principalement sur ses aspects proprement religieux (et cela est sans doute pour quelque chose dans certains doutes auxquels nous faisions allusion en commençant). Certes, les historiens les plus pénétrants y avaient bien décelé l'importance de la grammaire et de la logique, mais sans en faire un objet spécifique de leurs recherches. Depuis quelques décennies, et après les travaux précurseurs de $\mathbf{M}$. Grabmann ${ }^{56}$, H. Élie ${ }^{57}$, R. W. Hunt ${ }^{58}$, ou parallèlement à eux, les travaux consacrés à ce secteur se sont multipliés ; citons, sans prétendre être exhaustif : les éditions logiques d'Abélard, et d'autres logiciens du XIIe siècle, procurées par B. Geyer ${ }^{59}$, M. Dal Pra ${ }^{60}$, L. Minio-Paluello ${ }^{61}$, L. M. de

55. Anthony Kenny, Jan Pinborg, « Medieval Philosophical Literature ", in The Cambridge History..., op. cit. supra n. 54, p. 25.

56. En 1975 , le numéro 113 de la Revue internationale de philosophie a été dédié à cet historien éminent de la pensée médiévale ; on y lit des articles de cinq spécialistes des doctrines médiévales du langage : Lambert-Marie de Rijk, Desmond Paul Henry, Hubert Hubien, Jean Jolivet, Jan Pinborg.

57. Hubert Elie, Le Complexe significabile, Paris, Vrin, 1937.

58. R. W. HuNT, "Studies on Priscian in the Eleventh and Twelfth Centuries", Mediaeval and Renaissance Studies, I, 1941-1943, p. 194-231 ; II, 1950, p. 1-50.

59. Peter A aAelards Philosophische Schriften, «Beiträge zur Geschichte der Philosophie des Mittelalters ", Münster i. W., 1919-1933.

60. Pietro Abelardo, Scritti Filosofici, Rome/Milan, Edizioni di storia e letteratura, 1954.

61. Twelfth Century Logic, Texts and Studies, I et II, Rome, Edizioni di storia e letteratura, 1956 et 1958. 
Rijk ${ }^{62}$; les publications de plusieurs ouvrages de grammaire spéculative $^{63}$; des études qui attirent l'attention sur la structure linguistique de telle théologie ou ontologie ${ }^{64}$; un congrès de la Société internationale pour l'étude de la philosophie médiévale entièrement consacré à la question du langage et de la connaissance au Moyen Âge ${ }^{65}$; un volume collectif d'histoire de la philosophie médiévale entre 1100 et 1600 , dont la moitié des chapitres portent sur le langage et la logique et dont les éditeurs notent qu'on y a insisté sur " les matières jusqu'ici négligées par la littérature consacrée au Moyen Âge, et sur lesquelles la recherche récente a fait le plus de progrès ${ }^{66}$; la création par le regretté J. Pinborg et L. M. de Rijk, des "Symposiums européens de logique médiévale " ${ }^{67}$; les diverses études dues à ces deux spécialistes ${ }^{68}$... Ces publications, ces activités, et tant d'autres qu'on voudrait citer aussi, jettent une lumière neuve sur la philosophie du Moyen Âge ; certes, elles ont été suscitées, ou du moins environnées, par l'intérêt majeur qu'on porte aujourd'hui à la linguistique et généralement à la réflexion sur le langage. Mais si les chercheurs ont naturellement été incités à regarder le Moyen Âge sous cet angle, leur observation a rencontré tout autre chose qu'un artefact : l'ampleur des résultats obtenus et des perspectives ouvertes, et avant tout l'évidence de la chose même, attestent que la philosophie de cette époque avait bien fait des arts du langage l'instrument majeur de sa recherche, et en conséquence un de ses objets d'étude principaux. Nous voici donc en possession d'un nouveau critère pour déterminer les moments où commence et finit la philosophie médiévale. Quant à la fin, on sait

62. Petrus Abaelardus, Dialectica, Assen, Van Gorcum, 1956 ; Logica Modernorum, A Contribution to the History of Early Terminist Logic, I et II, Assen, Van Gorcum, 1962 et 1967 .

63. Ceux notamment des Daci : Boèce, Martin, Jean, Simon, publiés dans le « Corpus Philosophorum Danicorum Medii Aevi ".

64. Desmond Paul Henry, The Logic of Saint Anselm, Oxford, 1967 ; J. Jolivet, op. cit. supra n. 14 ; Alain DE LIBERA, Le Problème de l'êire chez Maître Eckhart : logique et métaphysique de l'analogie, Genève/Lausanne/Neuchâtel, Cahiers de la « Revue de théologie et de philosophie ", $1,1980$.

65. Sprache und Erkenntnis im Mittelalter, herausgegeben... unter der Leitung von Wolfgang KLUXEN, Berlin/New York, Walter de Gruyter, 1981 ( ( Miscellanea Mediaevalia », 13,1 et 2).

66. The Cambridge History of Later Medieval Philosophy, op. cit. supra n. 54 ; la phrase traduite ci-dessus est aux p. 2-3.

67. Ces colloques se tierınent depuis une quinzaine d'années ; il y a été beaucoup question de logique anglaise, mais aussi, par exemple, de Jean Buridan ; celui de 1965, à Poitiers, est centré sur la figure de Gilbert de la Porrée (Gilbert de Poitiers) : connu jusqu'ici comme théologien, on découvre son importance pour l'histoire de la logique.

68. J. PinborG, Die Entwicklung der Sprachtheorie im Mittelalter, "Beiträge zur Geschichte der Philosophie und Theologie im Mittelalter ", Münster i. W., XLII, 2, 1967 ; ID., Logik und Semantik im Mittelalter. Ein Überblick, Stuttgart/Bad Cannstatt, Frommann-Holzboog, 1972 ; ID., Medieval Semantics, Londres, Variorum Reprints, 1984. 
depuis longtemps que la nouveauté de la Renaissance a consisté pour une grande part, et peut-être d'abord, à faire passer la rhétorique, art de la persuasion et de la présentation des choses, avant la dialectique, art de la dispute et de la contrainte issue des enchaînements formels ; à considérer le langage dans son épaisseur concrète et son histoire ${ }^{69}$; parallèlement, la logique médiévale arrivait au bout de ses possibilités ${ }^{70}$. Ainsi les arts du langage changent de place dans le champ du savoir, entre eux et absolument, et cela définit une organisation nouvelle de la culture. Quant au début de la période, il se fait à plus petit bruit ; on peut, selon le critère qu'on a dit, le placer vers la fin du XI' siècle. C'est l'époque d'Anselme de Cantorbéry ; nées de la collatio monastique ses œuvres restent, sans préjudice de leur originalité profonde, apparentées aux modèles augustiniens ; mais, fait nouveau, il y inclut un dialogue De grammatico destiné à clarifier certaines difficultés des propositions attributives $^{71}$, et ses spéculations théologiques s'appuient très souvent sur la connaissance et la pratique du trivium ${ }^{72}$; c'est en tout cela justement qu'il se montre médiéval. Déjà, comme il arrivait à l'abbaye du Bec, le prieur Lanfranc soutenait contre Bérenger de Tours une controverse sur l'Eucharistie où l'un et l'autre maniaient la dialectique avec dextérité. Vers le même moment le problème posé au début de l'Isagogè subit une transformation significative : on ne se demande plus, avec Porphyre et Boèce lui-même, si les genres et les espèces sont des choses ou des conceptions de l'esprit, mais si ce sont des choses ou des mots ; la très fameuse « querelle des universaux » n'oppose pas d'abord, comme on le dit souvent, des platoniciens et des aristotéliciens (de fait), mais des logiciens qui, selon une formule intraduisible, commentent Porphyre soit in re, soit in voce, pratiquant ainsi un clivage nouveau dans la philosophie $^{73}$. C'est enfin le moment où, R. W. Hunt l'a montré, l'enseigne-

69. Voir par exemple Karl-Otto APEL, Die Idee der Sprache in der Tradition des Humanismus von Dante bis Vico, Bonn, Bouvier, Verlag Herbert Grundmann, 2 éd., 1975. Les critiques dirigées par les humanistes contre le langage technique des scolastiques médiévaux sont bien connues ; la transposition burlesque en est la harangue de Maître Janotus de Bragmardo dans RaBELAIS, Gargantua, chap. 19.

70. E. J. AshwORTH, in op. cit. supra n. 66 p. 795-796.

71. Édition : D. P. HENRY, The De Grammatico of St. Anselm, Notre Dame, Ind., Univ. of Notre Dame Press, 1964 ; voir du même, Commentary on De Grammatico, Dordrecht/Boston, Reidel, 1974.

72. Lothar STEIGER, « Contexe syllogismos. Über die Kunst und Bedeutung der Topik bei Anselm », Analecta Anselmiana, 1, 1969, p. 107-143.

73. On notera toutefois que les commentateurs alexandrins tardifs - Ammonius, David, Elias - avaient déjà appelé pēnte phōnaí, " les cinq voix », les prédicables étudiés par Porphyre ; mais ce n'est que dans des manuscrits grecs récents (XIV' et XV' siècles) que cette expression apparaît au titre de son ouvrage (Lorenzo Minio-Paluello, Aristoteles latinus, 1, 6-7, Bruges/Paris, Desclée De Brouwer, 1966, p. XIII). Au surplus, il est peu vraisemblable que les commentateurs cités plus haut aient été connus des Latins au XI' siècle. 
ment de la grammaire se charge de logique, d'une façon peut-être excessive et brouillonne, mais qui témoigne d'une nouvelle façon de considérer le langage ${ }^{74}$. Au-dessus de la couche néoplatonicienne qui sous-tend la philosophie du III' au XVI' siècle, le Moyen Âge occidental installe alors la structure qui lui est propre et qui durera quelque quatre cents ans : un ensemble de textes et de doctrines qui constituent une contribution à la logique et à la grammaire qui fut la plus dense de l'histoire peutêtre, une des plus denses à coup sûr ; et corrélativement un usage intense de leurs techniques dans tous les domaines du savoir ; renvoyons simplement sur ce point aux mille pages et plus du recueil déjà cité, Sprache und Erkenntnis im Mittelalter.

En deux mots donc, une première condition de la philosophie médiévale est réalisée par la naissance et l'installation du christianisme, la constitution des dogmes et la production des écrits théologiques des premiers siècles : ainsi se forme un contenu. Un autre pas est fait avec le changement de civilisation, qui donne à la spéculation une autre place dans l'espace social : elle devient le fait d'un groupe qui détient le pouvoir spirituel et, de façons diverses (collusion, substitution, relais), prend ainsi le caractère d'un pouvoir civil. Mais tout cela laisse inchangés les aspects techniques de la spéculation (les variations de niveau ne sont pas à considérer ici) : les genres et les méthodes, les grands thèmes et schèmes, sont généralement communs aux païens et aux chrétiens ; en ce sens, la pensée philosophique née dans le christianisme est encore un rameau de la philosophie antique, qui donne son dernier fruit, en Occident du moins, avec Jean Scot (IX' siècle). Cependant, les modifications dans le contenu et dans le soubassement historique et spirituel ne pouvaient pas rester sans conséquence sur l'exercice de la pensée : d'où sa nouvelle allure et sa véritable mutation, celle qui donne naissance à la philosophie/théologie médiévale. Elle consiste en l'apparition d'une méthode nouvelle qui affecte les genres, et, conservant en son fond le contenu religieux et philosophique de la doctrine chrétienne, lui donne une figure différente : c'est l'intégration à la recherche - et pas seulement à l'exposé des résultats - d'un usage réfléchi des arts du langage - grammaire, rhétorique, dialectique. Ce changement résulte d'une quantité de causes très diverses, qu'il est impossible d'énumérer ici, plus encore d'analyser ; citons la prégnance d'une culture religieuse fondée sur les textes, et la nécessaire herméneutique qui s'y accroche ; la perte du contact réel avec la science antique, qu'on ne connaît plus, pendant plusieurs siècles, que par des résumés livresques et abstraits ; la finalité purement administrative et religieuse du renouveau des études sous Charlemagne ; le carac-

74. Voir supra n. 58. 
tère spirituel et élitiste de la culture. Toutes ces causes font que l'essor social et intellectuel du XI' siècle ne trouve d'abord pour s'investir que les arts du trivium, ceux qui avaient été le mieux préservés parce qu'ils correspondaient le mieux aux besoins du Haut Moyen Âge. Ce changement donc s'amorce à l'époque carolingienne - citons Frédégise, Godescalc d'Orbais, le Jean Scot du De praedestinatione ${ }^{75}$; soutenu par la lecture exclusive, en matière philosophique, du début de l'Organon et des textes apparentés, qui mettent en valeur la profondeur et la complexité des rapports entre les choses, les pensées et les mots ${ }^{76}$, il n'aboutit vraiment que dans la seconde moitié du XIe siècle. À ce moment, la spéculation prend définitivement la forme qu'elle ne dépouillera qu'avec la Réforme et la Renaissance ; le retour à l'Antiquité auquel on assiste au XII' siècle - authentique, malgré la paucité des documents disponibles à l'époque - ne change en rien cette allure que la culture a prise, et qui la marque du véritable sceau médiéval.

\section{II. - DOUBLE LEVER EN ORIENT}

Les hommes peuvent être ennemis pour des raisons diverses : soit parce qu'ils exercent le même métier, ou qu'ils sont des voisins proches, ou de proches parents.

Al-Jāhiz, Le Livre des animaux.

Si le moment où commence la philosophie médiévale en Occident n'apparaît pas au premier coup d'œil, c'est parce que les premières spéculations chrétiennes se développent tout naturellement dans les formes littéraires et selon les schèmes théoriques qui étaient ceux de l'Antiquité. La technique philosophique antique persiste pour l'essentiel, mal-

\footnotetext{
75. FRÉdÉGIS, Epistola de nihilo et tenebris, PL 105, 751-756 ; C. GenNARo, Fridugiso di Tours e il " De substantia nihili et tenebrarum ", Padoue, Cedam, 1963 ; J. JoLJVET, Godescalc d'Orbais et la Trinité, Paris, Vrin, 1958 ; ID., "L'enjeu de la grammaire pour Godescalc ", in Jean Scot Erigène et l'histoire de la philosophie, Paris, Éd. du C.N.R.S., 1977, p. 79-87 ; JEAN SCOT, Liber de praedestinatione, PL 122, 347-440 ; voir aussi John MARENBon, From the Circle of Alcuin to the School of Auxerre, Cambridge, Cambridge University Press, 1981. La glose sur Porphyre étudiée et partiellement éditée par Victor Cousın, Fragments philosophiques. Philosophie scholastique, Paris, $2^{e}$ éd., 1840, p. 104-110, attribuée à Raban Maur par le manuscrit, paraít témoigner d'un état de la logique bien avancé pour le IXe siècle, on la verrait mieux de deux cents ans plus récente ; mais cette période est encore mal connue. $-\mathrm{V}$. Cousin s'est trompé en faisant consister toute la philosophie médiévale (ou " scholastique ", comme il dit) dans la querelle des universaux (ibid., p. 89), mais il a vu juste, malgré cette mauvaise raison, en la faisant commencer au $\mathrm{XI}^{*}$ siècle (p. 78 et suiv.).
}

76. Cela est bien marqué par Bruno MAIOLI, Gilberto Porretano : dalla grammatica speculativa alla metafisica del concreto, Rome, Bulzoni, 1979, p. 25-33. 
gré la différence des religions et des civilisations, jusqu'au début du second millénaire ; à ce moment-là seulement se constitue pleinement et durablement une nouvelle forme doctrinale avec sa structure et sa texture propres. Dans le monde de l'islam, les choses sont très différentes ${ }^{77}$. Mahomet communique son message à un peuple dont les relations avec le monde grec étaient ténues et purement pratiques. Quand elles deviendront plus étendues et plus étroites, ce seront d'abord des relations de puissance conquérante à peuples conquis et d'empire à empire. Or, pendant le même temps, l'activité intellectuelle a été intense au sein de la société musulmane ; notamment, le besoin de saisir au mieux le sens du Livre révélé et ses implications, celui de constituer un ensemble juridique complet et cohérent, et même les " retombées " spéculatives des controverses et conflits suscités par la question dú pouvoir (qui, et quel doit être le calife ?), ont suscité plusieurs disciplines dont l'objet peut être dit philosophique bien qu'elles soient toutes englobées dans la sphère religieuse : disciplines linguistiques, théologiques, juridiques. Elles constituaient ensemble un corpus déjà considérable au début du III $^{e}$ siècle de l'hégire, qui est le moment où commence à se développer, avec l'appui des souverains, une vaste entreprise de traductions d'œuvres scientifiques et philosophiques étrangères, et notamment grecques ; la fondation à Bagdad, en $217 \mathrm{H} / 832 \mathrm{C}$, de la « Maison de la sagesse " par le calife

77. La philosophie, au Moyen Âge, n'a pas parlé seulement le latin et l'arabe, mais aussi le grec et l'hébreu. Il conviendrait donc de poser à propos de la pensée hébraïque et de la byzantine les mêmes questions qu'on ne pose ici qu'à propos des Latins et des Arabes. Je ne me juge pas autorisé à le faire, n'ayant avec les deux domaines que j'ai dits que des rapports indirects, de seconde main. À un lecteur profane des ouvrages spécialisés, il apparaît que la pensée juive, après sa période hellénistique et celle où s'est constituée la dialectique talmudique, entre dans sa période médiévale avec Isaac Israeli et Saadia Gaon, morts l'un et l'autre vers le milieu du IX' siècle de l'ère chrétienne, soit au même moment que Fārābi. Le premier, élève de Kindi, assimile l'esprit de la falsafa ; le second, celui du kalām. Quant à la philosophie byzantine, elle prend si naturellement la suite de la philosophie grecque antérieure qu'il est difficile de marquer une césure où commencerait une ère médiévale. Longtemps confondue avec la théologie, elle s'en démarque toutefois et affirme son autonomie avec Michel Psellos (1018-1096; voir supra n. 44) et surtout Jean Italos (mort à la fin du $\mathrm{Xl}^{*}$ siècle), dont l'élève Eustrate de Nicée instaure une méthode de forme scolastique : voir Basile TATAKIS, "La philosophie grecque patristique et byzantine ", in Histoire de la philosophie, I, Paris, Gallimard ( "Encyclopédie de la Pléiade »), 1969, p. 997-1002. On pourrait donc proposer de dater de cette époque la philosophie byzantine médiévale ; mais le même auteur, en 1949, semblait désigner plutôt « l'aurore d'un nouvel humanisme " marqué par le nom de Photios : donc, le IX' siècle (La Philosophie byzantine, $2^{\mathrm{e}}$ supplément à l'Histoire de la philosophie d'Émile BrÉHIER, Paris, P.U.F., p. 129). I. P. Sheldon-Williams, quant à lui, attache une importance particulière aux spéculations sur les images, développées à partir, principalement, des vil' et $\mathrm{IX}^{\mathrm{C}}$ siècles (The Cambridge History..., op. cit. supra n. 15, p. 506 et suiv.). 
Al-Ma'mūn donne un point de repère symbolique et commode ${ }^{78}$. Cet apport rend possible l'apparition d'une nouvelle forme doctrinale, proche par sa méthode et ses concepts de la philosophie antique, et qui vient développer les connaissances que l'on avait des Grecs, satisfaire et attiser à la fois l'appétit spéculatif qui avait donné l'impulsion à ce développement inouï des contacts intellectuels. Ainsi la philosophie naît deux fois en islam : sous la forme d'abord d'une théologie originale, le kalàm ; sous celle ensuite d'un courant philosophique qui s'alimente pour une grande part aux sources grecques, la falsafa. Il n'y a aucune difficulté à dater assez exactement leurs origines respectives, on l'a vu ; il nous faudra seulement clarifier quelque peu la question de leur originalité par rapport à la philosophie grecque.

\section{Le kaläm.}

On ne voit guère au premier abord pourquoi le kalām, né de problèmes et de conditions purement arabes et islamiques, pourrait devoir quelque chose aux Grecs. En fait plusieurs choses, très diverses d'ailleurs, en ont fait naître l'hypothèse chez plusieurs historiens : la ressemblance entre telle doctrine soutenue par les auteurs du kalām (les mutakallimün) et telle théorie antique - ainsi, une conception atomistique de la matière ; le fait que le kaläm à un certain moment de son histoire a accueilli divers éléments de la philosophie hellénisante : on a pu penser que cela impliquait une connaturalité originelle ; les controverses très précoces avec les Chrétiens, qui supposent une dialectique commune ; l'obscurité où plongent encore les tout premiers débuts du kalām, et que certains des meilleurs travaux contemporains s'efforcent de dissiper ${ }^{79}$; il ne faut pas négliger non plus les habitudes mentales de certains historiens, qui, nourris de la philosophie grecque et la jugeant volontiers exclusive, en retrouvaient partout les concepts ${ }^{80}$; enfin, plusieurs auteurs musulmans

78. On n'entre pas dans le détail de tous ces travaux, ni des rapports entre les traductions syriaques et arabes des originaux grecs. Les histoires de la philosophie islamique comportent généralement des pages sur ce sujet, et des bibliographies ; par ex. : Henry Corbin, Histoire de la philosophie islamique, Paris, Gallimard ("Idées "), 1964, p. 30-40, 348-350 ; voir aussi Richard WALZER, Greek into Arabic, Oxford, Bruno Cassirer, 1963, et L'Éveil de la philosophie islamique, Paris, Geuthner, 1971, p. 21-42. Les travaux consacrés à ce sujet sont nombreux, et la matière est fort loin d'être épuisée.

79. On pense aux travaux de Josef VAN Ess, par exemple à l'ouvrage où il montre que la problématique du libre arbitre s'amorce déjà dans les traditions du Prophète et de ses compagnons (Zwischen Hadit und Theologie. Studien zum Entstehen prädestinatianischer Überlieferung, Berlin/New York, Walter de Gruyter, 1975).

80. Ainsi, Max HORTEN, Die philosophischen Systeme der spekulativen Theologen im Islam, Bonn, F. Cohen, 1912. 
anciens ont fait remonter très haut dans l'histoire du kalām le moment où il a intégré des éléments de la pensée grecque ${ }^{81}$. Rien de cela n'est décisif. Les textes auxquels on vient de faire allusion ont une intention polémique, du fait que (comme on le verra plus loin) la philosophie n'est pas en faveur auprès des théologiens sunnites, et qu'un moyen de déconsidérer des adversaires est d'affirmer qu'ils s'en sont inspirés ; les deux points qui précèdent sont inopérants ; les trois premiers ont un fondement historique, mais qui resterait à voir de près, en lui-même et dans les implications qu'on en tire. On ne peut entrer dans cette voie. On se contentera de l'exemple d'un livre récent.

Dans un gros ouvrage paru après sa mort, H. A. Wolfson s'est efforcé de donner une histoire du kalām dans ses relations avec la philosophie grecque, à laquelle selon lui les théologiens auraient emprunté leurs principales méthodes et problématiques; et cela, juste après le début du kaläm muctazilite ${ }^{82}$. Cette thèse n'emporte pas la conviction. Certes, on sait qu'avec et après Gazālī (Ve-XIe siècle), Rāzī (VIe-XIIe) ${ }^{83}$, des procédés et des concepts philosophiques sont partiellement intégrés au kalām ; on peut même voir apparaître chez le mu${ }^{c}$ tazilite Dirār, donc beaucoup plus tôt (fin II'-VIII' siècle), le mot māhiyya, "quiddité », qui témoigne d'une influence au moins ponctuelle de la philosophie grecque ${ }^{84}$; il serait de toute façon bien invraisemblable que les théologiens n'aient rien retenu de cette philosophie si largement répandue dans l'espace culturel. Mais on n'est pas fondé à supposer que cette influence fut aussi large, profonde et précoce que le prétend Wolfson. Sans entrer dans un examen détaillé de son ouvrage, on peut du moins considérer l'un de ses premiers arguments : pour leur interprétation des versets du Coran qui attribuent à Dieu une main, un visage, etc., les $\mathrm{Mu}^{\mathrm{c}}$ tazilites auraient mis en œuvre le concept aristotélicien d'analogie de proportion tel qu'il est défini dans l'Éthique à Nicomaque et la Métaphysique, alors que l'exégèse antérieure, non philosophique, consistait à simplement affirmer « une sim-

81. Al-Aș $\bar{c}^{\mathrm{C}} \mathrm{R} i$, Maqālät al-islamiyyīn, éd. Hellmut RITTER, Wiesbaden, Franz Steiner Verlag, $2^{e}$ éd., 1963, p. 483, 1.7 et suiv. : la doctrine des $\mathrm{Mu}^{c}$ tazilites sur la négation des attributs divins est reprise de " leurs frères, ceux qui s'adonnent à la philosophie "; ŚAHRASTĀNĩ, Kitāb al-milal wa-l-nihal, éd. WAKīl, Le Caire, 1387/1968, I, p. 29 : « les maîtres mu ${ }^{c}$ tazilites ont lu les livres grecs à l'époque de Ma'mūn et en ont mêlé les méthodes à celle du kalām "; voir aussi, dans un milieu autre et avec un éclairage différent, MAIMONIDE, Le Guide des égarés, trad. S. Munk, Paris, G. P. Maisonneuve et Larose, rééd., 1970 , I, p. 340 et suiv.

82. Harry Austryn Wolfson, The Philosophy of the Kalam, Cambridge, Mass., Harvard University Press, 1976.

83. Louis Gardet, M. M. ANawati, Introduction d̀ la théologie musulmane, Paris, Vrin, 1948, p. $72-73$, p. 35, et al.

84. AL-A ${ }^{C}$ ARİ, op. cit. supra n. 81, p. 281, 1. 7 : « Sa quiddité [mähiyyatuhu], c'est-àdire ce qu'Il est. " 
ple ressemblance (du type : un corps différent des corps) \ ${ }^{85}$. Les arguments accumulés par Wolfson portent peu : les textes où il pense trouver des preuves d'ordre soit historique, soit sémantique, sont disparates et surtout sont éloignés de la période qu'il considère. Rappelons encore que le reproche adressé aux Muctazilites par les hérésiographes musulmans : qu'ils s'inspirent des philosophes, peut toujours être polémique et relever davantage de l'amalgame que de l'analyse. Quant à l'application à l'exégèse coranique de l'analogie aristotélicienne, elle est déduite par Wolfson, non pas garantie par les textes. À prendre les choses telles qu'elles s'offrent, nous voyons ceci : les Muctazilites estiment par exemple que « les mains » de Dieu, dont parle le Coran, sont en fait « son pouvoir et sa bonté " ; or cette exégèse n'est rien d'autre que la simple interprétation d'une métaphore, sans contamination philosophique. A ̌šarì, qui la cite dans ses Maqālàt, ne met pas en cause à son propos un usage quelconque de la philosophie : « certains Muctazilites, dit-il, disent que la face de Dieu, c'est Dieu... ; que ses mains, cela signifie [ma cnähu] ses bienfaits " ${ }^{86}$. De telles formules se retrouvent chez Kindi (II'-III'/IX' siècle), philosophe et lié aux Muctazilites (ces deux points sont à noter), lorsqu'il cite et commente, justement pour expliquer une formule du Coran, les vers où le poète anté-islamique Imru' 1-Qays s'adresse à la nuit comme à un animal : « lorsque étirant le dos tu ramassais ta croupe vers un poitrail toujours plus lointain " ${ }^{87}$; il y a là, dit Kindi, " une façon de parler claire et usuelle dans la langue des Arabes ; elle leur est habituelle ; elle consiste à attribuer à une chose des traits qui ne sont pas dans sa nature »; le vers d'Imru' l-Qays « signifie [ $m a$ 'nähu] qu'il souhaite que le jour se lève ${ }^{88}$. Pas de philosophie là-dedans, rien qu'une explication du sens des mots, sans recours au concept d'analogie ; c'est une simple explication littéraire usant des mêmes formules qu'on lit dans le commentaire de Śaybānī ( $v^{e}$ siècle) au même vers : " cela signifie [ma 'nähu] qu'au moment où j'espérais que la nuit cesserait, elle ramassait sa croupe, c'est-à-dire qu'elle revenait ${ }^{89}$. - Cet excursus peut avoir paru long ; il fallait pourtant montrer sur un exemple précis qu'on ne doit pas se hâter de voir une influence grecque dans des écrits ou des

85. H. A. WolfSON, op. cit. supra n. 82, chap. I, 2.

86. AL-A $\breve{S}^{c}$ ARİ, op. cit. supra n. 81, p. $521,16-522,4$. Ces lignes se trouvent, on le notera, dans la partie de l'ouvrage où l'auteur est critique à l'égard des $\mathbf{M u}^{\mathbf{c}}$ tazilites et où il les accuse d'avoir une conception de Dieu imitée de celle des philosophes (cf. supra n. 81). 87. IMRU'L-QAYS, Mucallaqa, v. 45 ; in AL-ŠAYBĀNĪ, Šarh al-qașā'id al-'cašar, Le Caire, $2^{e}$ éd., 1384/1964, p. 100, trad. Jacques BERQUE, Les Dix grandes odes arabes de l'AntéIslam, Paris, Sindbad, 1979, p. 72.

88. Rasā'il al-KINDíal-falsafiyya, éd. Abū RìdA, I, Le Caire, 1369/1950, p. 375-376.

89. AL-Š́ $A$ YBĀNī, op. cit. supra n. 87. p. 100. 
idées qui relèvent simplement du fonds de la culture arabe et islamique la plus originelle ; en ce cas, de l'analyse littéraire. Précisément, ainsi que le note $\mathrm{R}$. Walzer, « quand les $\mathrm{Mu}^{\mathrm{c}}$ tazilites rencontraient des problèmes d'exégèse coranique, ils recouraient à l'interprétation des poètes anciens développée par les philologues contemporains " ${ }^{90}$. Aristote n'a rien à voir ici. Supposer que sa théorie de l'analogie sous-tend les recherches premières, ou quasi premières, de l'exégèse coranique, c'est supposer que l'interprétation des versets anthropomorphiques du Coran est d'emblée de l'ordre de l'attribution conceptuelle, autrement dit qu'elle concerne la représentation de la nature de Dieu ; or, comme l'indique J. Van Ess, la doctrine des attributs n'est pas, du moins au dẹbut, liée à celle de la nature divine, mais à celle des noms divins : les lignes directrices de la théologie islamique sont alors d'ordre linguistique ${ }^{91}$.

Bien entendu, d'autres instruments de pensée sont aussi utilisés dans le kalām : l'introspection quand on y raisonne sur la nature des actes humains ; l'imagination physique, avec la représentation des corps comme agrégats d'atomes... Mais l'importance du langage y reste primordiale (et on peut, sur ce point, faire le rapprochement avec la philosophie médiévale chrétienne) : outre l'intérêt porté à la langue par la culture arabe en général, l'islam se fonde sur un livre et sa théologie pourrait être considérée comme une vaste exégèse, puisqu'elle consiste finalement à déterminer et développer le sens des énoncés contenus dans le Coran en s'aidant de la grammaire, de la sémantique, de l'analyse des figures de style, aussi bien que de justifications et réfutations destinées à garantir cette interprétation. Un second caractère des traités de kalām est, en effet, leur allure dialectique : le bien-fondé des thèses qui y sont avancées n'est établi que si celles de quelque adversaire ont été entièrement réfutées : d'où cette « logique où l'adversaire est pourchassé à l'intérieur d'un réseau de questions n'ouvrant chaque fois que deux réponses, et rétrécissant progressivement les échappatoires " ${ }^{92}$. Si le kalām est, comme on l'a noté, une exégèse, il est aussi polémique et hérésiographique : aux écrits qui relèvent expressément de ce dernier genre, il faut en fait joindre tous les autres, qui, consacrés principalement à autre chose, ne font avancer leurs thèses que sur des chemins jonchés de leurs contradictoires. On peut trouver dans les écrits philosophiques grecs des pages remplies de discussions,

90. R. WALZER, Greek into Arabic, op. cit. supra n. 78, p. 182-183, avec renvoi à I. Goldziher. Cette remarque a d'autant plus de prix que $\mathrm{R}$. Walzer a tendance à majorer l'influence des Grecs sur la pensée arabe.

91. J. VAN EsS, "Sprache und religiöse Erkenntnis im Islam », in Sprache und Erkenntnis im Mittelalter, op. cit. supra n. 65, p. 216-235 (ici p. 234).

92. Guy Monnot, "Les doctrines des chrétiens dans le 'Moghni' de 'Abd al-Jabbār ", Mélanges de l'Institut dominicain d'études orientales, 16, 1983, p. 9-30 (ici p. 9). 
mais dans le kalām c'est autre chose, quant à la méthode et quant à la proportion.

Notre examen de la philosophie des chrétiens a laissé de côté son contenu. Toutefois, nous avions évoqué le thème de l'être, pour dire que son émergence dans le Moyen Âge latin n'était pas un commencement absolu : il avait déjà été posé par Porphyre, puis dans l'islam. Nous présenterons brièvement deux notions élaborées par les théologiens musulmans et qui sont, elles, sans antécédent à notre connaissance : celle des $a h w a ̄ l$, terme qu'on peut traduire par états, ou modes, et celle de l'inexistant. Le concept de "mode », élaboré par Abù Hāšim al-Gubbả'í (ob. 321/933), est destiné à résoudre, autant qu'il est possible, un des problèmes principaux de la théologie musulmane : comment concilier l'unité de Dieu avec ses attributs, sans ramener Dieu à une essence nue (ce que reproche $\mathrm{As̆}{ }^{c}$ arī aux $\mathrm{Mu}^{\mathrm{c}}$ tazilites), ni inversement poser des entités qui lui seraient coéternelles ? Le mode, dit Abū Hãsim au témoignage de Šahrastāni,

« est un attribut connaissable qui s'ajoute à l'être de Dieu comme essence et comme existant, mais qu'on ne connaît qu'avec l'essence et non isolément. Les modes sont des attributs ni existants ni inexistants, ni connaissables ni inconnaissables, c'est-à-dire qu'on ne les connaît qu'avec l'essence ${ }^{93}$.

Il ne paraît pas judicieux d'interpréter ce concept à l'aide des grilles aristotéliciennes et d'y voir, comme on l'a dit, « un substitut de la notion aristotélicienne de puissance » (à cause sans doute de la situation du mode : intermédiaire entre l'existence et l'inexistence), avec une « confusion du logique et de l'ontologique » et une « tendance à réifier les cadres logiques $"{ }^{94}$. L'élaboration de la notion de mode est précisément le fruit d'un effort pour " déréifier » la représentation qu'on peut se faire des attributs divins et briser l'alternative entre l'être et le non-être - ou, pourrait-on hasarder, le dégagement phénoménologique, à partir d'un cas particulier de visée religieuse, d'une région idéelle spécifique. Il faut mettre sous la même rubrique la constitution de la notion d'inexistant ( $m a{ }^{c} d u \bar{u}$ ), commencée dès le $\mathrm{II}^{\mathrm{c}}-\mathrm{III}^{\mathrm{c}} / \mathrm{IX}^{\mathrm{e}}$ siècle, que les $\mathrm{Mu}^{\mathrm{c}}$ tazilites ont spécialement développée et que les As̆carites refusent. Elle est destinée à rendre compte du fait que Dieu connaît des choses qu'il n'a encore créées ou qu'il ne créera jamais, sans leur supposer un mode d'être qui les lui ferait coéternelles ; comment il peut vouloir une chose et lui ordon-

93. Š SAHRASTĀNī, op. cit. supra n. 81, p. 82.

94. L. GARDET, M. M. ANAWATI, op. cit. supra n. 83, p. 326. 
ner « sois !" pour qu'elle existe, comme le dit le Coran ${ }^{95}$. Ici encore, il faut dépasser les oppositions classiques, sortir de l'ontologie aristotélicienne : ni la puissance ni la privation, qui sont localisées dans une substance, ne peuvent équivaloir au $m a^{c} d \bar{u} m$, qui précisément est hors de tout lieu, fût-il métaphysique. L'arrière-plan doctrinal en est tout aussi éloigné du platonisme et, notamment, pour rester dans le cas d'une philosophie religieuse, d'une théologie du Verbe où " était vie tout ce qui a été fait ", selon le prologue de l'Évangile de Jean.

\section{La falsafa.}

La première forme de la philosophie islamique se constitue donc, avec sa problématique et sa méthodologie propres, dès le II $/ \mathrm{VIII}$ e siècle ; elle donne ses premiers fruits spécifiques et spécifiquement philosophiques aux III ${ }^{e-I V e} / \mathrm{X}^{9}{ }^{96}$. Sa vie ne s'arrête certes pas là, mais, dès ce moment, le kalām a acquis sa pleine originalité, toute différente en son essence de la philosophie grecque. La seconde émergence, on l'a dit, est contemporaine d'un vaste effort en vue de rendre accessibles aux lecteurs de la langue arabe la science et la philosophie des Grecs : alors apparaît la falsafa, courant et mode de pensée significativement nommé par la simple transposition du mot grec philosophia. Or les philosophes - les faläsifa, pluriel de faylasüf, d'après philosophos : c'est le même procédé - héritent un ensemble complet de doctrines ; physique, morale, théologie, et sans même parler de la logique, les Grecs avaient traité de tout. Mais sur tous ces points, la religion et la théologie avaient aussi leurs doctrines, avec cette supériorité qu'elles n'étaient pas l'œuvre de païens. Les falāsifa devront donc faire pénétrer leurs disciplines dans un champ culturel déjà occupé ${ }^{97}$. Ils avaient par devers eux un important corpus

95. Voir sur ces points Annuaire de l'École pratique des hautes études (5e section), t. 84, p. $389-394 ;$ t. 85 , p. $381-384$.

96. Ceux qu'on a évoqués ici ne sont pas les seuls : il faudrait citer notamment les controverses sur le libre arbitre ; voir sur ce point Chikh Bouamrane, Le Problème de la liberté humaine dans la pensée musulmane (Solution Muctazilite), Paris, Vrin, 1978 ; Daniel Gimaret, Théories de l'acte humain en théologie musulmane, Paris/Louvain, Vrin/Peeters, 1980. On a préféré s'en tenir à ce qui sort des cadres de l'ontologie classique.

97. À vrai dire, elle ne se fera jamais vraiment sa place ; sauf peut-être chez les Shicites (voir les travaux d'Henry Corbin), les philosophes seront toujours, aux yeux des religieux, des gens douteux, bien qu'ils fussent assez souvent proches du pouvoir. Sur ces points, et sur la position difficile des philosophes entre la déviance et la conformité, voir Fehmi JADAANE, "Les conditions socio-culturelles de la philosophie islamique ", Studia Islamica, 38,1973 , p. 5-60. En Occident, on l'a dit, le changement de contenu religieux dans des formes conservées s'est fait comme naturellement : c'est pourquoi le mot philosophari a pu désigner la pratique de la sagesse chrétienne aussi bien que de la païenne : on mesure la différence sémantique avec falsafa. On le fait mieux encore si l'on considère la place que Šahrastānī et Ibn Hazm assignent aux philosophes, dans leurs revues respectives des 
d'œuvres antiques qui, pour dire les choses en gros, leur transmettait un aristotélisme néoplatonisant ; cette note particulière le rendait moins inassimilable à l'esprit d'un croyant que si ç'avait été un aristotélisme pur (malgré les formes qu'avaient su y découvrir et développer les néoplatoniciens précisément). Kindi commence sa Philosophie première par une introduction générale où il cite plusieurs textes d'Aristote et suggère que l'enseignement des philosophes est consonant à celui des prophètes ${ }^{98}$. Mais pour rassurer le calife Ma'mūn, le protecteur des traducteurs, il ne fallut pas moins qu'un rêve où Aristote en personne lui assura que la raison et la loi religieuse enseignaient la même morale ${ }^{99}$. Ašcari ne dut pas être favorisé de la même vision puisqu'il reprochait à tort ou à raison aux Muctazilites de s'être accointés aux philosophes. Du reste, l'attitude de Kindi à l'égard de la religion n'est nullement partagée par le philosophe et médecin Rảzì (IIle/IXe siècle) ni par Färäbỉ, son contemporain. Le premier se contente de la philosophie et va jusqu'à nier la prophétie ; le second fait peu de cas du kalām et, sur la question des rapports entre la religion et la philosophie, annonce Averroès : celle-ci, qui relève de la raison, est universelle et démonstrative, tandis que celle-là varie d'un peuple à l'autre. Dès le premier siècle donc de l'histoire de la falsafa (nous n'irons pas plus loin, comme pour le kalām), les positions sont prises et les deux lignes principales, qu'illustreront plus tard respectivement Avicenne et Averroès, sont amorcées ${ }^{100}$.

On peut maintenant se demander quel est, par rapport à la philosophie grecque, le degré d'originalité de la falsafa. Ce qu'en a connu le Moyen Âge latin en est entièrement dépendant, du moins aux yeux de ces lecteurs : Avicenne, Averroès, sont pour les Médiévaux des introducteurs à Aristote. Un savant contemporain, R. Walzer, déjà cité, et qui était venu à l'étude de la philosophie arabe à partir de celle de la grecque, a une tendance marquée à voir dans l'une une simple transposition de l'autre. Moins près de nous, Renan écrit que

« la philosophie, chez les Sémites, n'a jamais été qu'un emprunt purement extérieur et sans grande fécondité, une imitation de la philosophie grecque. Il en faut dire autant de la philosophie du Moyen Âge " ${ }^{101}$.

doctrines : le premier les met entre les Sabéens et les Arabes d'avant l'islam ; le second, entre ceux qui, pensant comme eux que le monde est éternel, ne lui reconnaissent pas de Créateur, et ceux qui pensent que le monde a commencé et a plusieurs créateurs.

98. Op. cit. supra n. 88, p. 102-104.

99. Al-Nadìm, Kitäab al-fihrist, èd. Réza TaGaddud, Téhéran, s.d., p. 303 ; Al-QıfTī, Ta'rîh al-hukamä', éd. Julius LiPPERT, Leipzig, 1903, p. 29.

100 . Voir J. JoliVET, "Le déploiement de la pensée philosophique dans ses rapports avec l'islam jusqu'à Avicenne ", in L'Islam, la philosophie et les sciences, Paris, UNESCO, 1981 , p. 35-65.

101. Ernest Renan, Averroès et l'averroïme, Paris, $2^{e}$ éd., s.d., p. VI. 
Laissons de côté la référence ethnographique qui relève de l'aspect le plus contestable de l'historiographie renanienne ; mais le rapprochement avec la philosophie médiévale latine doit nous retenir, d'autant que nous l'avons vue rester homogène à la philosophie grecque, jusqu'à la fin du $\mathrm{XI}^{\mathfrak{e}}$ siècle du moins. Qu'en est-il donc sur ce point de la falsafa ?

On a déjà noté qu'elle n'était pas née, comme la philosophie chrétienne, dans le milieu même (société, langue, culture) de la philosophie païenne ; elle ne résulte pas d'une germination naturelle, mais d'initiatives qui sont en quelque façon des ruptures avec une tradition bien établie, puisqu'il existait déjà dans le monde de l'islam des disciplines qui occupaient le champ de la philosophie, et que la religion musulmane n'est pas néoplatonicienne comme l'est la chrétienne. En outre, et cela va de soi après ce que l'on vient d'observer, les falāsifa, même quand ils ont été protégés par des princes, n'ont jamais exercé le pouvoir spirituel. Toutes ces conditions propres à la falsafa, et qui font sa différence originelle avec la philosophie élaborée par les chrétiens d'Occident, sont présentées d'une autre façon par Kindì dans le premier chapitre de sa Philosophie première. Exposant que la philosophie se construit progressivement et exige donc le concours de beaucoup d'hommes dans la suite des temps, il en infère qu'il faut chercher la vérité où qu'elle soit, même chez des philosophes d'autres nations et qui parlent une autre langue, l'adapter au temps et la faire parler arabe ${ }^{102}$; et, de suite, il critique violemment les religieux, ennemis de la philosophie, et qu'il faut, dit-il, chasser des chaires qu'ils ont usurpées et occupent indûment ${ }^{103}$.

Ainsi le premier des falāsifa revendique hautement pour la philosophie arabe la place que lui assigne Renan ; mais, et cela donne son vrai sens à son dire et le met très loin de celui de l'historien français, il la conçoit et la projette comme le contraire même d'une répétition : ce doit être à la fois pour lui une reprise et un prolongement, un progrès. Considérée dans sa réalisation effective, son œuvre philosophique, du moins ce qui nous en reste, le loge tout naturellement dans la tradition de la philosophie antique, mariant, pour le dire en bref, des schèmes et des thèmes propres au néoplatonisme au réseau conceptuel constitué par Aristote ; il s'y joint un effort pour concilier tout cela avec la religion musulmane, puisque, rappelons-le, selon lui les prophètes et les philosophes ont une même théologie et une même morale ${ }^{104}$. C'est ainsi qu'il éla-

102. 'alā mağrà 'àdati l-lisān wa-sunnati l-zamān.

103. Op. cit. supra n. 88 , p. 102-104; le passage sur la croissance du savoir reprend des passages d'Aristote, Métaphysique, $\alpha, 1$.

104. Ibid., p. 105. 
bore une démonstration de la non-éternité du monde ${ }^{105}$. On peut dire que sur la voie qu'il ouvre ainsi les falāsifa ultérieurs s'engageront tous. Certes, leurs doctrines ne sont pas identiques entre elles, l'étendue et la profondeur de leurs œuvres diffèrent. Un Färābì, un Avicenne, sont des géants ; un Averroès, un très grand esprit. Cela dit, il faut reprendre à leur propos ce qu'on a constaté des premiers philosophes chrétiens, jusqu'à Jean Scot y compris : ce sont des néoplatoniciens, leur philosophie reprend et continue celle des païens en l'unissant à une religion nouvelle. Cependant, le schème de la chaîne d'or nous permet de préciser une différence déjà indiquée entre les philosophes du christianisme, qui pouvaient l'intégrer sans difficulté à la représentation du monde postulée par leur feligion, et ceux de l'islam. Chez ces derniers, le schème (sans l'image explicite) se retrouve : par exemple chez Kindi qui, dans son Epitre sur l'Agent vrai, décrit un univers où des agents " au sens impropre du mot " transmettent tout au long de leur hiérarchie un " être-agi " (infi'a $l$ ) dont la première cause est l'action véritable de Dieu ${ }^{106}$; chez Fãrābi, qui expose la façon dont à partir du Premier viennent à l'être successivement les intellects et les corps célestes ${ }^{107}$, et Avicenne reprendra cette doctrine, tout en la modifiant ; etc. ${ }^{108}$. L'intérêt de la chose n'est pas tellement dans la prégnance de cette représentation, qui témoigne simplement de la persistance du néoplatonisme ; le plus important pour nous ici est qu'elle représente la structure d'un univers régi par des lois et où, surtout, même la causalité divine passe à travers des relais : or les théologiens musulmans sont unanimes à affirmer que Dieu agit directement sur chacune de ses créatures. Le désaccord est ici le même, sous une autre forme, que pour la morale : pour Fāräbī, elle est chose rationnellement nécessaire et démontrable, et non décret divin enseigné dans un Livre révélé ${ }^{109}$.

105. Ibid., p. 114-122 et 201-207. Cette démonstration qui va contre la doctrine d'Aristote n'en utilise pas moins des méthodes philosophiques : la situation est ici voisine de celle de Jean Philopon, philosophe chrétien d'Alexandrie, établissant contre Proclus la non-éternité du monde.

106. Ibid., p. 182-184.

107. Kitäb arā' ahl al-madinat al-fädila, éd. Albert NADER, Beyrouth, 1953, p. 44-45.

108. Voir notamment l'aspect qu'a pris ce schème, combiné à des représentations d'origine zoroastrienne, dans la philosophie de Suhrawardi : H. CoRsin, En Islam iranien, aspects spirituels et philosophiques. T. II : Sohrawardi et les platoniciens de Perse, Paris, Gallimard ("Bibliothèque des idées »), 1971, p. 119-126; Sohraward, L'Archange empourpré, quinze traités et récits mystiques, trad. du persan et de l'arabe par $\mathrm{H}$. CorBIN, Paris, Fayard, 1976, p. 31-89.

109. Les $\mathbf{M u}^{\mathrm{c}}$ tazilites pensent que les commandements divins sont rationnels : ce point est l'un de ceux où ils se rapprochent clairement des philosophes. 
Ainsi les rapports entre la philosophie et la religion sont beaucoup plus tendus dans l'islam que dans le christianisme, bien que la plupart des philosophes aient cherché à unir l'une et l'autre ; et même, il n'est pas sans exemple que la kalām ait influé sur la falsafa : la doctrine avicennienne de l'essence a été préparée par les controverses théologiques sur l'inexistant ${ }^{110}$. Ces relations complexes entre les deux formes prises par la philosophie en Islam valent d'être méditées, au terme d'une étude qui a surtout porté sur des successions d'une part, sur des différences et des coupures de l'autre, dans chacun des domaines que nous avons considérés. Et, pour en revenir au jugement de Renan sur la falsafa, on doit donc reconnaître à celle-ci la fécondité qu'il lui refuse, tout en constatant avec lui qu'elle a été importée dans le monde islamique, à la différence de ce qui s'est passé dans le cas de la philosophie médiévale chrétienne. Sont-elles l'une et l'autre une " imitation » de la grecque ? Il est plus juste de dire qu'elles en sont la continuation, qu'elles en ont prolongé l'existence dans des conditions autres, jusqu'à ce que la falsafa s'éteigne, du moins dans la partie occidentale du monde de l'Islam, et que l'Europe chrétienne se donne une forme de pensée nouvelle qui constitue proprement la philosophie médiévale latine, homologue donc en quelque façon au kalām, de nature et d'allure tout autres cependant.

Jean JOLIVET, École pratique des hautes études (5e section).

110. Voir supra n. 12. 